\title{
An Image-Based Inertial Impact (IBII) Test for Tungsten Carbide Cermets
}

\author{
L. Fletcher ${ }^{1}(\mathbb{1}) \cdot$ F. Pierron ${ }^{1}$
}

Received: 20 June 2018 / Accepted: 10 August 2018 / Published online: 24 August 2018

(c) The Author(s) 2018

\begin{abstract}
Testing tungsten carbide cermets at high strain rates is difficult due to their high stiffness and brittle failure mode. Therefore, the aim of this study is to apply the image-based inertial impact (IBII) test methodology to analyse the high strain rate properties of tungsten carbide cermets. The IBII test uses an edge on impact test configuration with a narrow stress pulse. The narrow input pulse travels through the specimen in compression and reflects in tension causing failure. Full-field measurements of acceleration and strain are then coupled with the virtual fields method to identify the stiffness components and tensile strength of a test sample at high strain rates. Image deformation simulations were used to select optimal test processing parameters and predict the associated experimental errors. The elastic modulus and tensile strength of the tested tungsten carbide cermet samples were successfully identified using the IBII test at strain rates on the order of $1000 \mathrm{~s}^{-1}$. No significant strain rate dependence was detected for either the stiffness or tensile strength.
\end{abstract}

Keywords Tungsten carbide $\cdot$ High strain rate $\cdot$ Full-field measurement $\cdot$ Tensile strength $\cdot$ Virtual fields method

\section{Introduction}

Tungsten carbide cermets are used in a wide range of applications in which they undergo high strain rate loading. The high wear resistance, stiffness, strength and fracture toughness make tungsten carbide cermets ideal for use in machine tooling [1]. In order to adequately model machining processes and effectively design new machining tools the high strain rate properties of tungsten carbide cermets need to be fully characterised. Unfortunately, experimental difficulties limit the available data for the high strain rate properties of tungsten carbide cermets.

Traditionally, the high strain rate properties of ceramic materials are analysed in compression using the split Hopkinson pressure bar (SHPB) technique [2-4]. The SHPB method uses a small sample sandwiched between two long bars which act as load cells. A stress wave is imparted onto the specimen through the bars and the readings on the strain

$\triangle$ L. Fletcher

1.c.fletcher@soton.ac.uk

F. Pierron

f.pierron@soton.ac.uk

1 Engineering and the Environment, University of Southampton, Southampton, Hampshire SO17 1BJ, UK gauges are used to infer the properties of the test sample. A key assumption required to analyse the strain gauge data from SHPB is that the test specimen must be in a state of quasi-static equilibrium. In order to satisfy this assumption specimens must be small relative to the overall bar length such that the stress waves reverberate and quickly damp out in the test sample. These inertial effects generally occur in the initial (i.e. elastic) portion of the test. Therefore, it is generally accepted that the SHPB technique cannot be used to measure the elastic modulus of materials at high strain rates since failure/damage occurs before quasti-static equilibrium is reached, and therefore only an apparent modulus can be obtained [5].

For brittle materials the problems associated with inertial effects are exacerbated. The small strain observed prior to failure in brittle materials means that the specimen can fail before the inertial effects have damped out, corrupting not only the stiffness but also the strength measurements [3]. Another assumption required by the SHPB analysis is that the specimen and the bars remain in contact and planar with respect to one another. Given the high stiffness of tungsten carbide this can be a difficult constraint to satisfy without modifying the bars using end caps. However, modifications of the bars using end caps can lead to increased problems with inertial effects and 
dispersion due to the impedance mismatch between the bars and the end caps $[3,6]$.

To the authors best knowledge there is only a single study investigating the strain rate dependence of the strength of tungsten carbide/cobalt cermets in the open literature [6]. The study by Mandel et al. [6] analysed the strain rate dependence on the compressive strength of tungsten carbide/cobalt cermets using a specially modified SHPB technique. The results of this study showed that the compressive strength exhibited an exponential relationship with respect to strain rate from quasi-static rates to strain rates of $500 \mathrm{~s}^{-1}$. Unfortunately, data for the elastic properties and tensile strength of tungsten carbide cermets is unavailable at high strain rates.

Recently, new high strain rate testing techniques have been developed that do not rely on the assumptions that limit traditional SHPB analysis [7-9]. The Image-Based Inertial Impact (IBII) test is a viable alternative to the SHPB and has been successfully applied to the analysis of quasi-brittle materials such as composites $[8,10]$ and brittle materials such as concrete [11-13]. The IBII technique takes advantage of ultrahigh speed $(>1 \mathrm{MHz})$ camera technology to perform full-field displacement measurements during a dynamic test in the wave propagation regime. From the kinematic fields the material properties can be identified using an inverse technique such as the virtual fields method (VFM). The IBII test method has significant potential for investigating the properties of brittle materials such as tungsten carbide and will allow for the measurement of material properties at high strain rates that are not currently obtainable using the standard SHPB technique.

The aim of this study is to develop an IBII test method to investigate the stiffness and tensile strength of tungsten carbide (WC) cermets at high strain rates. The first section of this paper outlines the concept and required theory for the IBII test. Following this, the second section briefly details the design of the test components using explicit dynamics simulations. This then leads to the third section which outlines the image deformation simulations used to validate the stiffness identification procedure and select optimal smoothing parameters. The fourth section describes the experimental results obtained with the IBII test for several different tungsten carbide cermets. This includes identification of the elastic modulus, Poisson's ratio and the tensile strength. The final sections of this paper discuss the limitations and future applications of the IBII test along with a summary of the main findings.

\section{Test Concept and Theory}

\section{The Imaged-Based Inertial Impact (IBII) Test}

A simplified schematic of the IBII test configuration is shown in Fig. 1. The specimen of dimensions $L_{S} \times H_{S} \times t_{S}$ is loaded by a short compressive pulse, $F(t)$ by impact with a projectile. A compressive stress wave then travels through the specimen, reflects and becomes tensile. The input pulse is selected such that failure occurs once the wave reflects off of the free edge in tension. Throughout the propagation of the stress wave fullfield displacement measurements are obtained through white light imaging and image processing. From this the strain and acceleration fields are derived. These kinematic fields are then used with the methods described in the following sections to determine the material properties.

\section{VFM}

A detailed discussion of the VFM applied to the IBII test is provided in references $[8,10]$. Therefore, only the concept and the main results will be briefly revisited taking into account the differences that are specific to the material being tested. Note that a detailed discussion of the VFM formulation can be found in reference [14].

Consider the dynamically impacted sample shown in Fig. 1. Suppose that kinematic full-field measurements are taken over the surface of the specimen for a specified time interval. The desired output from the test is the constitutive behaviour of the material being tested. In order to relate the constitutive behaviour of the material to the kinematic fields the principle of virtual work is used. The process is referred to as the VFM. If body forces are neglected, the principle of virtual work is given by:

$-\int_{V} \boldsymbol{\sigma}: \boldsymbol{\epsilon}^{*} d V+\int_{\delta V} \boldsymbol{T} \cdot \boldsymbol{u}^{*} d S=\int_{V} \rho \boldsymbol{a} \cdot \boldsymbol{u}^{*} d V$

where $V$ is the volume of the specimen and $\delta V$ is the surface of the volume. The virtual displacement field is $\boldsymbol{u}^{*}$ and the virtual strain tensor (derived from $\boldsymbol{u}^{*}$ ) is $\boldsymbol{\epsilon}^{*} . \boldsymbol{\sigma}$ is the Cauchy

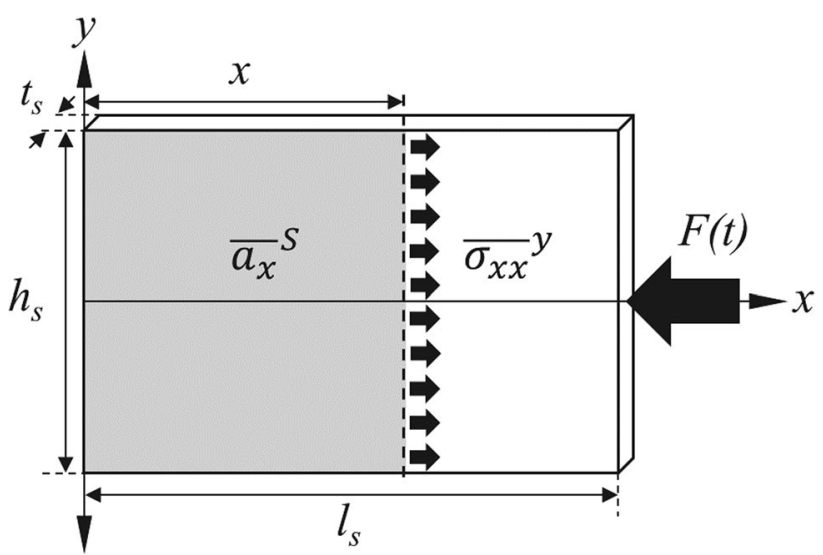

Fig. 1 IBII test schematic. Note that the variables ${\overline{\sigma_{x x}}}^{y}$ and ${\overline{a_{x}}}^{S}$ correspond to Eq. (9) 
stress tensor and $\boldsymbol{T}$ is the Cauchy stress vector acting on the boundary surface $\delta V$. The material density is given by $\rho$ and the acceleration field is $\boldsymbol{a}$. The matrix dot product is given by : and vector dot products is denoted by $\cdot$. The first term of Eq. (1) is referred to as the internal virtual work, the second term is the external virtual work and the third term is the acceleration virtual work. All kinematic fields in this equation are functions of both time and space. In order to simplify the notation in the following sections, the time and space variables as function notation have been omitted.

The VFM uses the principle of virtual work to generate equilibrium equations that relate the stress components to accelerations and externally applied forces. If the stress components are substituted with an appropriate constitutive law then the VFM can be used to directly identify material properties from full-field measurements. The only condition that must be satisfied is that the virtual displacement vectors are continuous and piecewise differentiable. Additionally, $\boldsymbol{u}^{*}$ has to be 'VFM-admissible' so that no unwanted unknowns appear in the equation (e.g. the distribution of pressure from a projectile impacting the edge of a sample).

\section{Constitutive Model and Assumptions}

In order to identify material parameters using Eq. (1) it is necessary to substitute the stress tensor with a constitutive model. Here, an isotropic linear elastic constitutive law is used. This constitutive law is described by the following equation, using the plane stress assumption:

$\left[\begin{array}{c}\sigma_{x x} \\ \sigma_{y y} \\ \sigma_{x y}\end{array}\right]=\left[\begin{array}{ccc}Q_{x x} & Q_{x y} & 0 \\ Q_{x y} & Q_{x x} & 0 \\ 0 & 0 & \frac{Q_{x x}}{2}\end{array}\right]\left[\begin{array}{l}\epsilon_{x x} \\ \epsilon_{y y} \\ \epsilon_{x y}\end{array}\right]$

where $Q_{x x}$ and $Q_{x y}$ are the stiffness components given by:

$Q_{x x}=\frac{E}{1-v^{2}} ; Q_{x y}=v Q_{x x}$

where $E$ is the elastic modulus and $v$ is Poisson's ratio.

This constitutive law can now be substituted into the principle of virtual work (Eq. 1). Here, a number of simplifying assumptions are made: (1) the density, thickness and material properties of the specimen do not vary in space; (2) the kinematic fields are uniform through the thickness; and (3) the specimen is in a state of plane stress. If these assumptions are applied then the principle of virtual work becomes:

$$
\begin{aligned}
&-Q_{x x} \int_{S}\left(\epsilon_{x x} \epsilon_{x x}^{*}+\epsilon_{y y} \epsilon_{y y}^{*}\right.\left.+\frac{1}{2} \epsilon_{x y} \epsilon_{x y}^{*}\right) d S \\
&-Q_{x y} \int_{S}\left(\epsilon_{x x} \epsilon_{y y}^{*}+\epsilon_{y y} \epsilon_{x x}^{*}-\frac{1}{2} \epsilon_{x y} \epsilon_{x y}^{*}\right) d S+ \\
& \int_{l} \boldsymbol{T} \cdot \boldsymbol{u}^{*} d l=\rho \int_{S} \boldsymbol{a} \cdot \boldsymbol{u}^{*} d S
\end{aligned}
$$

where $S$ is the surface of the specimen and $l$ is the perimeter of the specimen. In order to use Eq. (4) for stiffness identification two virtual fields are required that generate independent equations for $Q_{x x}$ and $Q_{x y}$. This will be described in the following section.

\section{Virtual Fields for Stiffness Identification}

In this section, several virtual fields are described that will be used for stiffness identification. Here, the objective is to identify the stiffness components $Q_{x x}$ and $Q_{x y}$ which are related to the elastic modulus and Poisson's ratio. In the following sections, overline notation will be used to denote spatial averaging. For example: ${\overline{\sigma_{x x}}}^{y}$ denotes the average stress over the line at $x$ shown in Fig. 1. Additionally, $\bar{a}_{x}^{S}$ denotes the surface average of the acceleration between the considered line and the free edge (shaded area in Fig. 1).

\section{Virtual Field Set 1: Manual}

This set of virtual fields is denoted as 'manual' as the virtual fields are manually specified. The derivation of manual virtual fields for a similar loading condition is described in [8]. Therefore, the full derivation will be omitted. The first virtual field to be used is as follows:

$\left\{\begin{array}{l}u_{x}^{*}=l_{s}-x \\ u_{y}^{*}=0\end{array}\left\{\begin{array}{l}\epsilon_{x x}^{*}=-1 \\ \epsilon_{y y}^{*}=0 \\ \epsilon_{x y}^{*}=0\end{array}\right.\right.$

It is generally difficult to accurately measure the impact force in a dynamic test. Thus, the virtual field in Eq. (5) has been selected to cancel the contribution of the impact force at $x=l_{s}$ (see Fig. 1). Therefore the external virtual work term in Eq. (4) is zero and the desired stiffness parameters are only a function of the measured strain and acceleration fields. Substituting the virtual field in Eq. (5) into Eq. (4) gives:

$Q_{x x}{\overline{\epsilon_{x x}}}^{S}+Q_{x y}{\overline{\epsilon_{y y}}}^{S}=\rho{\overline{a_{x}\left(l_{s}-x\right)}}^{S}$ 
where ${\overline{\epsilon_{x x}}}^{S}$ and ${\overline{\epsilon_{y y}}}^{S}$ are the surface average of the strain fields over the whole field of view. $\overline{a_{x}\left(l_{s}-x\right)}$ is the surface average of the acceleration over the whole field of view weighted by the function $l_{s}-x$. As there are two unknown stiffness components an additional virtual field is required to obtain another equation that relates the measured kinematic fields to the stiffness components. This can be obtained from the following virtual field:

$\left\{\begin{array}{l}u_{x}^{*}=0 \\ u_{y}^{*}=\frac{\left(l_{s}-x\right) y}{h_{s}}\end{array}\left\{\begin{array}{l}\epsilon_{x x}^{*}=0 \\ \epsilon_{y y}^{*}=\frac{\left(l_{s}-x\right)}{h_{s}} \\ \epsilon_{x y}^{*}=-\frac{y}{h_{s}}\end{array}\right.\right.$

Again, this virtual field has been selected to cancel the contribution of the impact force. Substituting the virtual field in Eq. (7) into Eq. (4) gives:

$$
\begin{gathered}
-Q_{x x}\left[\frac{\overline{\left(l_{s}-x\right) \epsilon_{y y}}}{h_{s}}-\frac{\overline{y \epsilon_{x y}}}{h_{s}}\right]-Q_{x y}\left[\frac{\overline{\left(l_{s}-x\right) \epsilon_{x x}}}{h_{s}}+\frac{\overline{y \epsilon_{x y}}}{h_{s}}\right] \\
=\rho \frac{\overline{\left(l_{s}-x\right) y a_{y}}}{h_{s}}
\end{gathered}
$$

Therefore, at any time step Eqs. (6 and 8) can be solved simultaneously to obtain the stiffness components $Q_{x x}$ and $Q_{x y}$.

\section{Virtual Field Set 2: Special Optimised}

An infinity of virtual fields can be substituted into Eq. (4) to generate equations for the stiffness components. Therefore, the question of how to select these virtual fields arises. For a perfect data set (i.e. no noise) all sets of independent virtual fields will give the correct results for both stiffness components. In practice, full-field measurements contain noise. Thus, it is desirable to automatically select virtual fields that reduce the effects of noise. These virtual fields are known as 'special optimised'. The derivation of the special optimised virtual fields for the dynamic case is essentially the same as [15]. The main difference is that the external virtual work term is replaced by the acceleration virtual work term. The interested reader is also referred to [14] for a detailed description of the formulation of special optimised virtual fields. Here, the virtual fields will be expanded using bilinear piece wise functions similar to Q4 finite elements.

\section{Virtual Field Set 3: Stress-Gauge Equation}

As described in $[8,10]$ the rigid body virtual field $u_{x}^{*}=1$, $u_{y}^{*}=0$ can be used to derive the stress-gauge equation. The stress-gauge equation is given as follows: ${\overline{\sigma_{x x}}}^{y}=\rho x{\overline{a_{x}}}^{S}$

where ${\overline{\sigma_{x x}}}^{y}$ is the average stress over the line at $x, \rho$ is the material density and $\vec{a}_{x} S$ is the area averaged acceleration from the free edge to the line at $x_{0}$. These quantities are shown schematically in Fig. 1. This equation can also be used for stiffness identification. Consider the linear elastic isotropic constitutive law described in Eq. (2). The ' $x$ ' direction stress component is related to the stiffness $Q_{x x}$ as follows:

$\sigma_{x x}=Q_{x x}\left(\epsilon_{x x}+v \epsilon_{y y}\right)$

Therefore, by linearly fitting the average stress ${\overline{\sigma_{x x}}}^{y}$ against the average strain ${\overline{\epsilon_{x x}+v \epsilon_{y y}}}^{y}$ the stiffness component $Q_{x x}$ is obtained. In order to use this method to determine $Q_{x x}$ the Poisson's ratio can be obtained using either of the first two virtual field sets described above.

\section{Virtual Fields for Strength Identification}

Rigid body virtual fields can be used to derive equations that relate stress averages to averages of the acceleration fields. These equations can be used for strength identification, as in [10]. For the present study the linear stress gauge equation will be used for strength identification. The linear stressgauge equation is given as follows:

$\sigma_{x x}(L S G)=\rho x \bar{a}_{x}^{S}+\frac{12 \rho x y}{h_{s}^{2}}\left({\overline{a_{x} y}}^{S}-\overline{a_{y} x} S+x \overline{a_{y}} S\right)$

Here the first term in the equation is the stress-gauge equation (Eq. 9). The second term of this equation describes the first moment of the axial stress in terms of weighted averages of the acceleration components. The linear stress-gauge equation has been previously used for tensile strength identification using the IBII test on composite materials. Note that the full derivation for this relationship is given in "Appendix" and in [10].

\section{Numerical Test Design}

The procedure for designing an IBII test using a parametric design sweep has been outlined in detail in reference [10]. Therefore, only a brief description of the procedure will be given here along with the results that are relevant to the specific material being tested.

\section{Model Configuration and Design Sweep Criteria}

The objective of the design sweep is to determine the experimental configuration required to achieve the desired reflected tensile stress and cause failure in the test sample. 
An estimate of the compressive and tensile strengths of tungsten carbide is needed to set design limits on the input pulse required for the test. For tungsten carbide cermets the compressive strength is on the order of $3 \mathrm{GPa}$ and is expected to increase with strain rate $[6,16]$. Therefore, the maximum magnitude of the input pulse can be conservatively set at $3 \mathrm{GPa}$ in compression.

Data on the quasi-static tensile strength of tungsten carbide is limited as ceramics are extremely sensitive to test machine misalignment. The strength of ceramics are also dependent on defect distributions, exhibiting a volume effect which is normally characterised using Weibull statistics. Due to these experimental difficulties ceramics are normally tested in bending to give the transverse rupture strength which can be used to obtain the Weibull modulus. Thus, in a similar manner to [17] it is possible to relate the tensile strength to the transverse rupture strength as a function of the Weibull modulus. The Weibull modulus for tungsten carbide with $13 \%$ cobalt binder can be taken as $\sim 7[18,19]$. Given this Weibull modulus and assuming the samples are tested in three point bending the tensile strength is approximately half of the transverse rupture strength. Depending on binder content the transverse rupture strength of tungsten carbide cermets varies from 1 to $3 \mathrm{GPa}$ giving an estimated tensile strength between 0.5 to $1.5 \mathrm{GPa}[16,19,20]$. Unfortunately, it is not known if the tensile strength of tungsten carbide cermets changes with strain rate, making it difficult to determine the magnitude of the input pulse required to cause tensile failure. The failure modes for tungsten carbide in quasi-static compression and tension are significantly different, in compression coarse grained tungsten carbide exhibits plastic deformation while in bending it is linear elastic to failure $[16,18$, 19]. Thus, care must be taken using this data to infer a strain rate dependence on the tensile strength when designing the IBII test for tungsten carbide. Therefore, a tensile strength of $\sim 1 \mathrm{GPa}$ at high strain rates will be assumed for the design sweep. This gives a design envelope for the input compressive stress of less than $3 \mathrm{GPa}$ with a reflected tensile stress of greater than $1 \mathrm{GPa}$.

The components required to implement the IBII test are shown schematically in Fig. 2. Several of these parameters are fixed based on experimental constraints. The gas-gun used for the experiments has a $50 \mathrm{~mm}$ bore and can launch projectiles with a diameter up to $45 \mathrm{~mm}$ encased in a $50 \mathrm{~mm}$ diameter sabot. For simplicity, the height of the projectile and waveguide was fixed at $45 \mathrm{~mm}\left(h_{p}=h_{w g}=45 \mathrm{~mm}\right)$.

The tungsten carbide samples that were obtained had dimensions of $60 \times 30 \times 4 \mathrm{~mm}$, fixing the length and height of the specimen $\left(l_{s}=60 \mathrm{~mm}\right.$ and $\left.h_{s}=30 \mathrm{~mm}\right)$. Previous work had shown that the waveguide length does not affect the result of the design sweep as long as it is at least twice the projectile length [10]. For the IBII test a short pulse

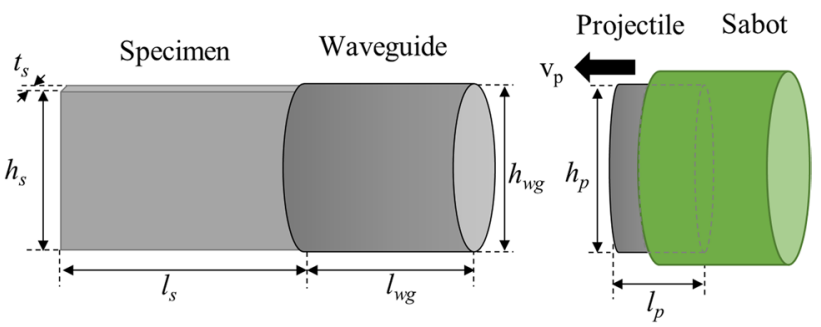

Fig. 2 Schematic of the physical components for the image-based inertial impact test experiments

is required. As such, the projectile length will be less than the specimen length (given that the specimen and the waveguide have similar bulk wave speeds). Thus, the waveguide length has been fixed at the same length as the specimen $l_{w g}=60 \mathrm{~mm}$.

A design sweep of the projectile length and projectile velocity was conducted to achieve the desired tensile stress. The projectile length was swept from 5 to $25 \mathrm{~mm}$ in $2.5 \mathrm{~mm}$ increments. The projectile velocity was swept from 10 to $70 \mathrm{~m} \mathrm{~s}^{-1}$ in $10 \mathrm{~m} \mathrm{~s}^{-1}$ increments. The material selected for the projectile and waveguide was steel. The reason for this is that steel alloys with a yield stress on the order of $1 \mathrm{GPa}$ are readily available and cost effective. Having a high yield stress projectile allows a $1 \mathrm{GPa}$ pulse to be imparted on the tungsten carbide sample without significantly yielding the projectile and clipping the input pulse.

\section{Finite Element Model}

All explicit dynamics models were constructed using ANSYS APDL LS-DYNA (v16.2). Simulations were conducted in two dimensions using PLANE162 elements with the plane stress assumption (4-node, reduced integration). The waveguide, projectile and sabot are cylindrical in the experiments but were modelled as two dimensional components for the purpose of the design sweep. This approach reduces computation time and provides suitable design predictions as described in [10].

In order to select appropriate simulation parameters a parametric sweep of time step, beta (stiffness proportional) damping and mesh size was conducted. These parameters were selected such that the error between the mean stress calculated from the acceleration (see Eq. 9) and the mean stress extracted directly from the FE model was below $1 \%$. The selected simulation parameters are summarised in Table 1. The data output step was selected to simulate the camera frame rate used in the experiments (5 Mfps giving a data output step of $0.2 \mu \mathrm{s})$. The total simulation time was set such that the stress wave would travel through the sample, reflect and return to the contact point with the waveguide. The default automatically generated two-dimensional 
Table 1 Finite element simulation parameters and material properties

Simulation parameters

\begin{tabular}{llll}
\hline Element type & PLANE162 & & \\
Mesh size & $0.5 \mathrm{~mm}$ & & \\
Nominal time step & $0.7 t_{\text {crit }}$ & & \\
Beta damping & 0.1 & & \\
Data output step & $0.2 \mu \mathrm{s}$ & & Nylon \\
\hline Material properties & Tungsten carbide & Steel & 1140 \\
\hline$\rho\left(\mathrm{kg} \mathrm{m}^{-3}\right)$ & 14500 & 7800 & 3.45 \\
$E(\mathrm{GPa})$ & 550 & 210 & 0.4 \\
$\nu$ & 0.24 & 0.29 & \\
\hline
\end{tabular}

contact algorithm in LS-DYNA was used to model contact between all components. All materials were modelled as linear elastic and isotropic. The material properties for the various components used in the simulations are summarised in Table 1.

\section{Parametric Design Sweep Results}

The parametric sweep results are summarised in Figs. 3 and 4. The peak mean tensile stress and compressive stress as function of projectile length and velocity is shown in Fig. 3. All cases tested have an input compressive stress below the predicted compressive strength of $3 \mathrm{GPa}$. Based on maximising the ratio of the input compressive stress to reflected tensile stress (see Fig. 4) a projectile length of $L_{P}=10 \mathrm{~mm}$ or $12.5 \mathrm{~mm}$ will be optimal. For these two projectile lengths an impact speed between of $V_{P}=50$ to $70 \mathrm{~m} \mathrm{~s}^{-1}$ will give a reflected tensile stress of $\sim 1 \mathrm{GPa}$ or greater. For the experiments the projectile length was set to $10 \mathrm{~mm}$ as a similar design sweep showed that this length would also be suitable for testing other ceramics such as silicon or boron carbide.

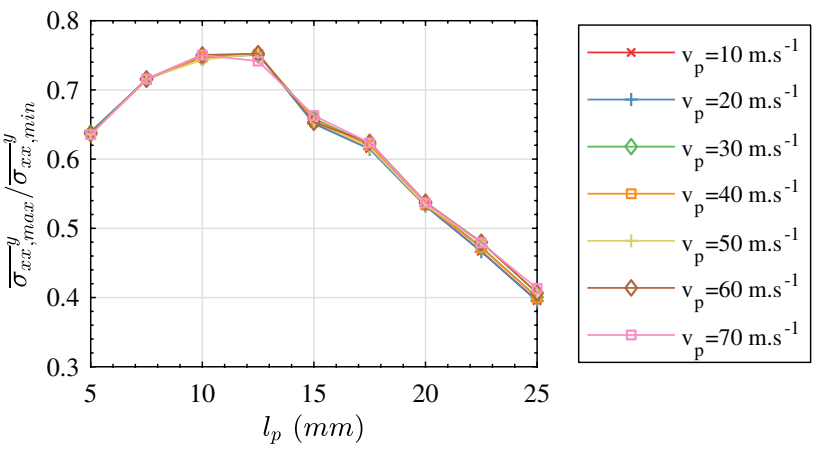

Fig. 4 Ratio of maximum average tensile stress to input compressive stress predicted by the finite element model

\section{Experimental Methodology}

\section{Tungsten Carbide Samples}

Four different tungsten carbide cermets were obtained from a commercial vendor, General Carbides. The specific grades of tungsten carbide tested here are the same as in [21]. The composition and relevant properties for the tested materials are summarised in Table 2 . The specimens were machined by the vendor to nominal dimensions of 60 $\times 30 \times 4 \mathrm{~mm}$. Hereafter the different grades of tungsten carbide will be referred to using the specimen identifier given in Table 2. Different specimens from each grade will be referred to using a numeral preceding identifier. For example: 1-WC-F-Ni6 refers to specimen number one from the tungsten carbide with fine grains and $6 \%$ nickel binder.

\section{Impact Apparatus}

The IBII tests were conducted on a custom built impact rig at the University of Southampton. An annotated photograph of the impact chamber is shown in Fig. 5(a) along with close up views of the projectile (b), waveguide and specimen (c). The projectile and waveguide were machined
Fig. 3 a Maximum average tensile and $\mathbf{b}$ compressive stress predicted by the finite element model as a function of projectile length $\left(l_{p}\right)$ and projectile velocity $\left(v_{p}\right)$
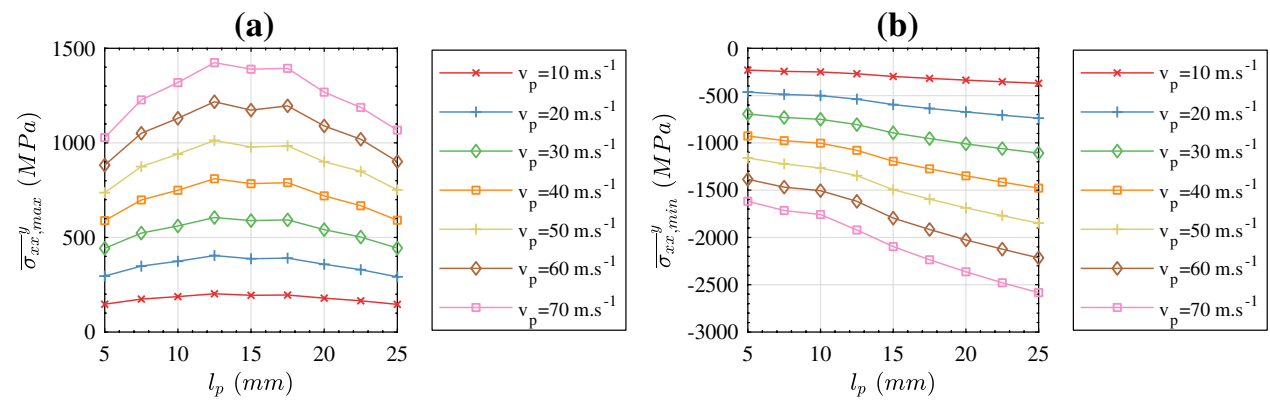
Table 2 Properties of the four tungsten carbide cermets tested in this study, see [21]

\begin{tabular}{llllll}
\hline Specimen identifier & Manufact. number & Binder material & $\begin{array}{l}\text { Binder } \\
\text { content } \\
(\%)\end{array}$ & $\begin{array}{l}\text { Average } \\
\text { grain size } \\
(\mu \mathrm{m})\end{array}$ & Density $\left(\mathrm{kg} \mathrm{m}^{-3}\right)$ \\
\hline WC-F-6Ni & GCN-061 & Nickel & 6.8 & $0.5-1.0$ & $14.54 \pm 0.04$ \\
WC-F-6Co & GC-206 & Cobalt & 6.7 & $1.0-1.5$ & $14.77 \pm 0.06$ \\
WC-F-13Co & GC-313 & Cobalt & 12.5 & $0.75-1.25$ & $13.97 \pm 0.03$ \\
WC-C-13Co & GC-613CT & Cobalt & 12.8 & $2.0-3.0$ & $13.69 \pm 0.01$ \\
\hline
\end{tabular}

(a)

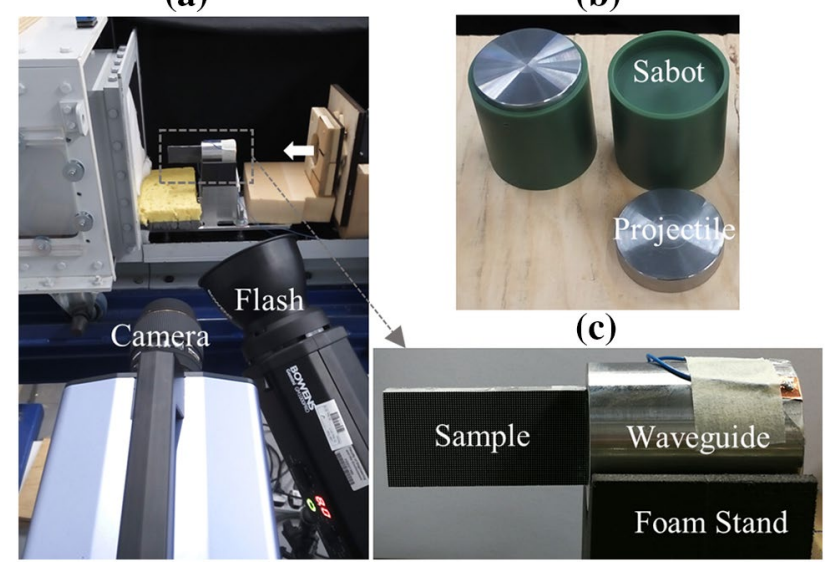

Fig. 5 a Photograph of the test section and capture chamber showing the camera, flash and mounted specimen. b Projectile and sabot assembly. c Close up view of the test sample attached to the waveguide

from a cylindrical rod of high strength steel alloy 15CDV6. This material was selected as its quasi-static yield stress is $993 \mathrm{GPa}$. The specimen was bonded to the waveguide using cyanoacrylate glue. The waveguide was mounted in front of the exit of the gas gun barrel using a wedge shaped foam stand.

The camera was triggered using a contact trigger consisting of two strips of copper tape on the front of the waveguide. When the projectile impacts the waveguide the circuit is completed and the camera is triggered. A delay of $12.4 \mu$ s was used before recording to account for the time taken for the wave to propagate through the waveguide. Two infra-red light gates at the barrel exit were used to measure the projectile speed. The photographic flash used for lighting has a rise time of approximately $100 \mu$ s so it could not be triggered using the same trigger as the camera. In order to trigger the flash the light gates were connected with a custom Arduino system. The Arduino system automatically calculated the projectile speed and the time to impact given the range to the target. The Arduino then automatically triggered the flash allowing for the rise
Table 3 Imaging and full-field measurement parameters

\begin{tabular}{ll}
\hline Camera & Shimadzu HPV-X \\
Pixel array size & $400 \times 250$ \\
Total frames & 128 \\
Inter frame time & $0.2 \mu \mathrm{s}$ \\
Integration (shutter) time & $0.11 \mu \mathrm{s}$ \\
Grid pitch & $0.7 \mathrm{~mm}$ \\
Grid sampling & 5 pixels $/$ period \\
Field of view & $56 \times 35 \mathrm{~mm}$
\end{tabular}

Note that measurement resolution and smoothing parameters are summarised in Table 4

time. The gas gun pressure chamber was set for a nominal impact speed of $50-55 \mathrm{~m} \mathrm{~s}^{-1}$.

\section{Imaging and Full-Field Measurement Setup}

All experimental data were collected using a Shimadzu HPV-X ultra high speed camera coupled with a Sigma $105 \mathrm{~mm}$ macro lens. The grid method was used for all fullfield measurements as it offers a better compromise between spatial and deformation resolutions than digital image correlation [22]. This is especially important given the small pixel array size of the camera used for the experiments. Obviously, the drawback of the main grid method is the need to apply a regular grid pattern to the sample. Grids with a $0.7 \mathrm{~mm}$ pitch were bonded to the test specimens using the process outlined in [23]. Relevant imaging and full-field measurement parameters are summarised in Table 3.

When using ultra-high speed cameras such as the Shimadzu HPV-X it is important to consider the effects of the low pixel fill-factor. When using the grid method the low pixel fill-factor leads to parasitic fringes in the displacement fields $[24,25]$. In [24, 25] it was shown that slightly blurring the image by defocusing the lens mitigated the effects of the low pixel fill-factor. Here, the blurring was assessed by using an out-of-plane movement test prior to the dynamic test as described in [10]. 


\section{Experimental Data Processing}

The raw grey-level images were processed using an open source code, as described in [26]. A detailed review of the grid method including experimental applications is also provided in reference [26]. Here, the grid method will only be briefly described.

Unlike digital image correlation which uses a nominally random pattern, the grid method uses a periodical (and nominally sinusoidal) pattern. Signal processing algorithms based on Fourier transforms are then used to determine the phase at each pixel of the grid sampled by the camera. The phase can then be directly related to the displacement on the samples surface. When the in-plane displacement is large, discontinuities can occur in the phase maps. This can be corrected for using a suitable spatial unwrapping algorithm. The spatial unwrapping algorithm used here was developed in [27] and comes with the open source code described in [26]. Discontinuities in the phase can also occur in time when the rigid body motion of the sample is large compared to the pitch of the grid used. For the IBII test configuration considered here the rigid body motion between frames is small compared to the grid pitch and it increases monotonically over the test. Therefore, the discontinuities in time can be corrected by adding integer multiples of $2 \pi$ to the phase fields to ensure that the average displacement over the field of view increases monotonically over the test duration. The temporal unwrapping code used here was developed in-house. After extracting the displacement fields using the grid method Matlab code from [26] all further processing was conducted using a custom post-processing Matlab program (v R2017a).

As the displacement fields contain noise it is necessary to smooth in space and time prior to numerical differentiation. The spatial filter used here was Gaussian with a kernel of $41 \times 41$ pixels. The temporal filter used here was a third order Savitsky-Golay filter over 11 frames. This raises the question of filter kernel selection for optimal identification. This issue is addressed in the following section using an image deformation software pipeline to simulate the experiment (see "Image Deformation Simulations"). The strain and acceleration fields were then derived from the displacement fields. Note that smoothing was only applied directly to the displacement field prior to numerical differentiation. In order obtain the acceleration fields, the velocity fields were derived first using a centred finite difference method. The velocity field was then differentiated again in the same manner to obtain the acceleration fields with no additional smoothing. The strain fields were obtained from the displacement fields using a centred finite difference method. Forward and backwards differences were applied at the edges of the data. The kinematic fields were then used with procedures described in "Test Concept and Theory" section to identify the stiffness components and tensile strength.

Another consideration with full-field measurement techniques is how to deal with the missing data on the borders of the specimen. For the grid method one pitch of data is lost along the border in the direction of the respective displacement component (e.g. one pitch on each edge in the $x$ direction is lost for the $x$ displacement with no pitches lost in the $y$ direction). It has been previously shown that padding to replace the missing data significantly improved identification with the VFM [28]. Here, a similar padding method was adopted. The specific method used for padding was to linearly extrapolate the displacements in space by fitting the last five data points along the edge. Note that it is only necessary to pad the $x$ displacement field in the $x$ direction by one pitch on each edge. Similarly, the $y$ displacement field is only padded in the $y$ direction. The raw images, data processing program and associated output are provided for all tested specimens in the data repository detailed at the end of the manuscript.

\section{Image Deformation Simulations}

A software pipeline was developed that simulates the imaging process in the IBII test. The purpose of this procedure was to simulate the effects of camera noise and limited spatial resolution to select smoothing parameters for the experimental data in a rational manner.

\section{Image Deformation Procedure}

The general methodology used in this study is similar to previous work using simulated experiments to analyse imaging procedures [12, 13, 28-32]. For image deformation simulations finite element displacement fields are used to numerically deform images. These images can then be processed using exactly the same procedure as the experimental images. The advantage of using finite element displacement fields is that the underlying constitutive parameters are known and serve as a reference value for error analysis.

The displacements fields were extracted from the explicit dynamics model of the optimal configuration selected in "Parametric Design Sweep Results" section. These fields were then used to synthetically deform grid images using the analytical description of a sinusoidal grid pattern:

$G(x, y)=2^{b}\left[I_{0}+\frac{\gamma}{4}\left(1+\cos \left(\frac{2 \pi x}{p}\right)\right)\left(1+\cos \left(\frac{2 \pi y}{p}\right)\right)\right]$

where $G(x, y)$ is the grey level value at the point $(x, y), b$ is the bit range of the camera (for the HPV-X $b=10$ ), $I_{0}$ is the average illumination, $\gamma$ is the image contrast and $p$ is the 
pitch of the grid pattern. The parameters of the simulated grid images were set to replicate the experimental case (grid pitch of $0.7 \mathrm{~mm}$ sampled at 5 pixels/period). The contrast of the experimental images was assessed using ImageJ (i.e. the overall percentage of the dynamic range used between light and dark areas of the grid image). A typical grid image in this study was found to use $50 \%$ of the dynamic range after blurring. The contrast of the simulated grid images was set to replicate the observed contrast and average illumination of the experimental images.

Gaussian white noise was then added to the simulated images before they were processed using the same custom built Matlab program as was used for processing the experimental data. The grey level noise was evaluated from an experimental static image sequence and the standard deviation of the grey level noise was expressed as a percentage of the full dynamic range. This was found to average $0.35 \%$ over a static experimental grid image sequence. Therefore, a uniform grey-level noise was applied to the images with a standard deviation of $0.35 \%$ of the dynamic range. An (a)

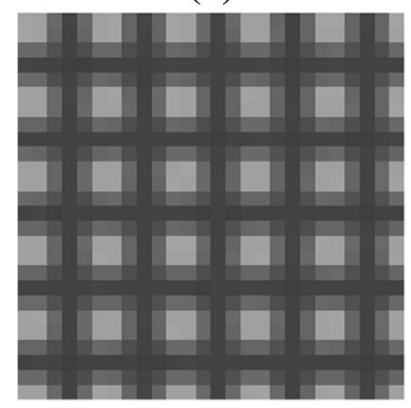

(b)

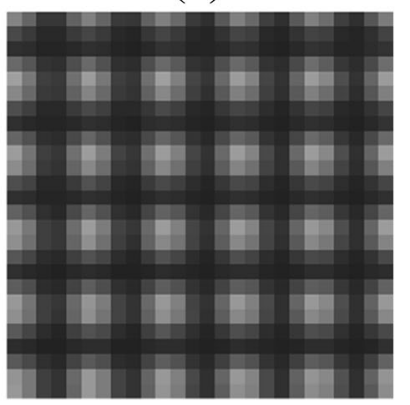

Fig. 6 a Synthetic grid image and $\mathbf{b}$ experimental grid image example synthetic grid image is shown in Fig. 6 next to an experimental grid image. Note that the sequence of synthetic deformed grid images (without noise) is supplied in the data repository detailed at the end of the manuscript.

\section{Image Deformation Stiffness Identification}

To illustrate the stiffness identification procedures a single case from the image deformation sweep will be considered here. The Gaussian filter window was set to $41 \times 41$ pixels and the Savitsky-Golay filter was third order applied over 11 frames. Note that in the following section it will be demonstrated that these filter parameters give a near optimal trade-off between random and systematic error (see "Image Deformation Error Analysis" section). As these are the same smoothing parameters as used to process the experimental data the stiffness identification presented here will serve as a useful comparison to the experimental results presented in "Stiffness Identification" section. The kinematic fields for this simulated specimen are not shown here but can be found in the data repository detailed at the end of the manuscript.

A sensitivity study was conducted to determine the appropriate virtual mesh density for use with the special optimised virtual field routine. The identification was found to converge with a virtual mesh density of $5 \times 3$ elements $(x, y)$ so this was used for all subsequent processing with the optimised virtual fields method. The identification with the optimised virtual fields procedure is shown in Fig. 7 for the stiffness components $Q_{x x}$ and $Q_{x y}$. Figure 7 shows that both methods are not stable in the early portion of the test with both methods stabilising at approximately $3.5 \mu \mathrm{s}$ for $Q_{x x}$. The reason for this is that there must enough information encoded in the kinematic fields such that the stiffness can be identified (i.e. acceleration and strain). This only occurs
Fig. 7 Stiffness identification for the synthetic image deformation using the manual and optimised virtual fields procedures. a Identification of $Q_{x x}$ for and $\mathbf{b} Q_{x y}$ (a)

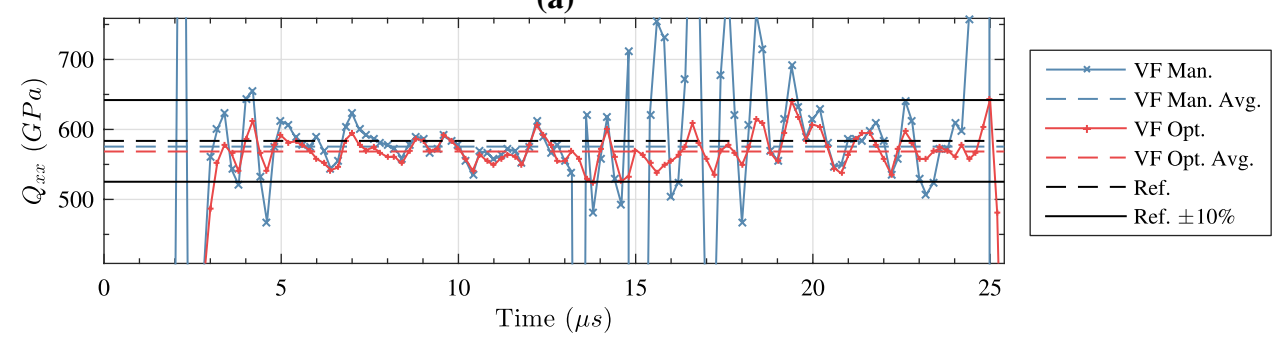

(b)

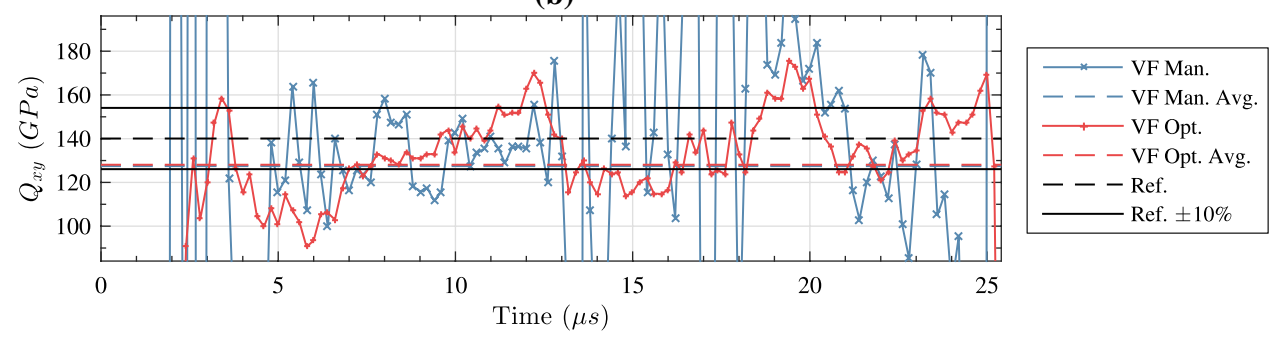


once a significant portion of the stress wave has entered the specimen. Figure 7 also shows that it is much harder to identify $Q_{x y}$ than $Q_{x x}$. This result was expected as the deformation is predominantly axial with small lateral strains and acceleration. Furthermore, the Poisson's ratio for tungsten carbide is relatively small.

There are also significant differences between the manual and optimised virtual fields routines. In general, the optimised virtual field routine is much more stable. This is because the optimised virtual fields are able to follow areas of high strain signal minimising the effects of noise. This is particularly noticeable when the wave reflects off the free edge at around $15 \mu$ s where the manual virtual fields show a period of extreme instability. Therefore, only the optimised virtual fields will be considered for further analysis in the rest of this paper.

A single stiffness value can be obtained from the optimised virtual field method by averaging the response over the frames for which the identification is stable. Only the compressive loading portion is considered for the VFM analysis. The reason for this is that in the experiments the specimen will damage and fail in tension corrupting the modulus identification with the VFM. Thus, the response can averaged from 5 to $13 \mu$ s to give a single identified stiffness. For the optimised virtual fields the following stiffness components are identified for the image deformation case considered here, $Q_{x x}=568 \mathrm{GPa}$ and $Q_{x y}=128 \mathrm{GPa}$. This can be compared to the target values input into the finite element model, $Q_{x x}=584 \mathrm{GPa}$ and $Q_{x y}=140 \mathrm{GPa}$. There is a notable difference between the identified stiffness components and the target values which can be attributed to a combination of systematic (i.e. camera spatial and temporal resolution) and random (i.e. camera noise) errors . In order to separate and predict these two error sources multiple iterations of noise need to be considered as described in the following section.

Stress-strain curves were constructed at all axial slices along the specimen length using the stress-gauge equation. Several representative stress-strain curves along the specimen

(a)

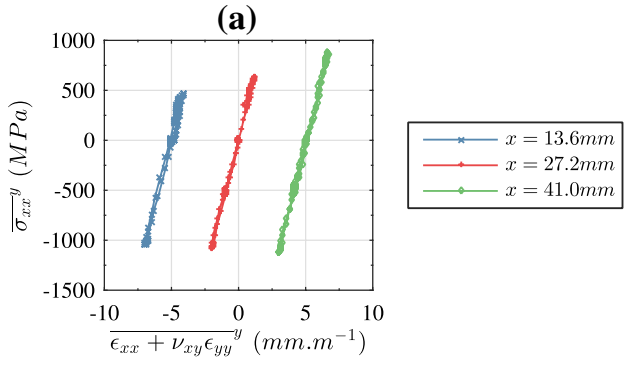

Fig. 8 Stiffness identification for the synthetic image deformation using the stress-gauge equation. a Stress-strain curves at several locations along the specimen length, note that all curves pass through the length are shown in Fig. 8a. For every section along the length the stress-strain curve can be linearly fitted to obtain the stiffness component $Q_{x x}$. As mentioned previously, in the experiments the specimen will fail after the wave reflects therefore only the compressive loading portion of the stress-strain curve is linearly fitted to obtain the stiffness. The identified stiffness along the specimen length is shown in Fig. 8b. A single identified stiffness can then be obtained by averaging over the middle $50 \%$ of the specimen to avoid edge effects from the Gaussian smoothing filter. For this case the mean identified stiffness over the length is $Q_{x x}=582 \mathrm{GPa}$ compared to a target value of $Q_{x x}=584 \mathrm{GPa}$.

This section has demonstrated the stiffness identification procedure for a single copy of noise and a single smoothing filter. In order to select the optimal smoothing parameters and predict the experimental error multiple copies of noise must be considered for several different smoothing kernels. The following section outlines this procedure.

\section{Image Deformation Error Analysis}

Various smoothing kernels were swept for the spatial and temporal filters. The spatial filter selected was Gaussian and the kernel was swept from 0 (i.e. no smoothing) to a kernel of 71 pixels in increments of 10 pixels $\left(S_{k}=0,11,21, \ldots, 71\right)$. The temporal filter selected was a third order Savitsky-Golay filter which was swept from a kernel of 0 to 25 frames in increments of 5 frames $\left(T_{k}=0,5,10, \ldots, 25\right)$. Note that both filters require that the specified kernel size be an odd number. For each combination of spatial and temporal smoothing kernels thirty iterations of noise were processed. The stiffness components $Q_{x x}$ and $Q_{x y}$ were then identified using the procedures outlined in "Image Deformation Stiffness Identification" section. The mean of the identified stiffness components for the thirty iterations was compared to the stiffness input into the finite element model to assess the systematic error. Specifically, the systematic error $E r r_{s y s}$ was defined as follows:

$\operatorname{Err}_{s y s}=\frac{\left(\overline{Q_{i j, I D}}-Q_{i j, F E}\right)}{Q_{i j, F E}}, i j=[x x, x y]$

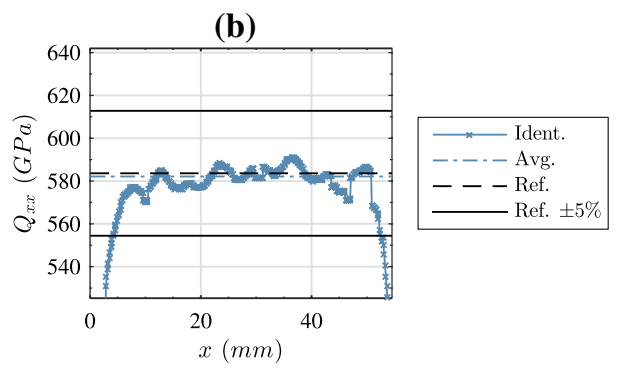

origin but have been offset by $5 \mathrm{~mm} \mathrm{~m}^{-1}$ for clarity. b Identified stiffness $Q_{x x}$ as function of axial position from the free end at $x=0$ 
where $\overline{Q_{i j, I D}}$ is the mean stiffness identified from the image deformation simulation over thirty iterations of noise and $Q_{i j, F E}$ is the target stiffness input into the finite element model. The random error was defined as the standard deviation of the identified stiffness over the thirty iterations of noise normalised by the target stiffness value:

$\operatorname{Err}_{\text {rand }}=\frac{1}{Q_{i j, F E}} \sqrt{\frac{\sum_{k=1}^{N}\left(Q_{i j, I D}^{k}-\overline{Q_{i j, I D}}\right)^{2}}{N-1}}, i j=[x x, x y]$

where $N$ is the number of iterations of noise $(N=30)$ and $Q_{i j, I D}^{k}$ is the stiffness identified with $k$ th copy of noise. These two measures were combined to form the total error $E r r_{\text {tot }}$ as follows:
$E r r_{\text {tot }}=\max \left(\left|E r r_{\text {sys }} \pm 2 E r r_{\text {rand }}\right|\right)$

The systematic, random and total errors for the identification of $Q_{x x}$ and $Q_{x y}$ using the special optimised virtual fields is shown in Fig. 9. The error analysis for $Q_{x x}$ identified with the stress-gauge equation is shown in Fig. 10. From Figs. 9 and 10 the systematic error tends to increase with increased temporal smoothing and decrease with increased spatial smoothing. The local minima for the systematic errors in Figs. 9 and 10 occur with significant spatial smoothing and minimal temporal smoothing with the stiffness parameters being systematically under predicted. Normally, it is expected that the minimum systematic error occurs with minimum smoothing for both the spatial and temporal filters. This suggests that
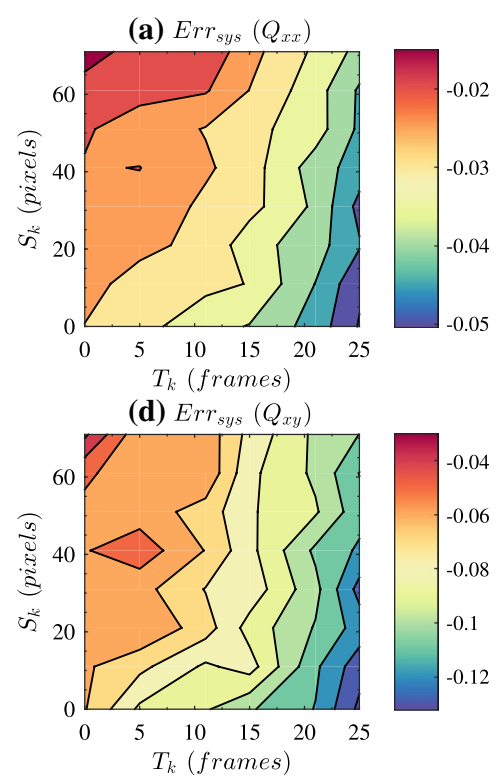
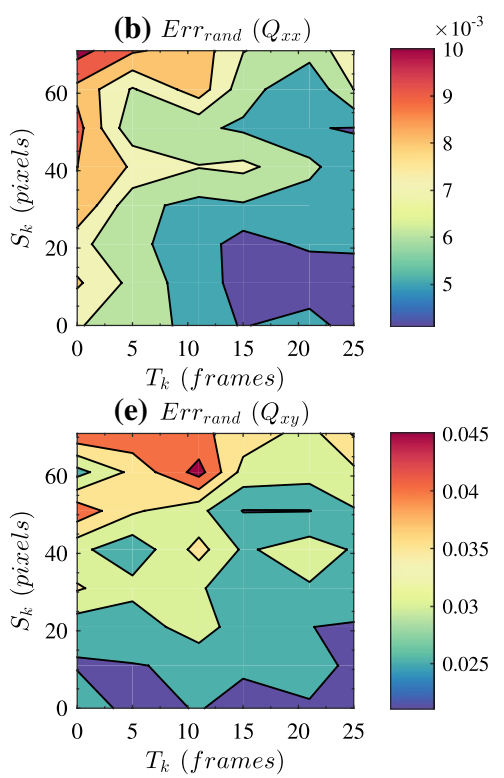
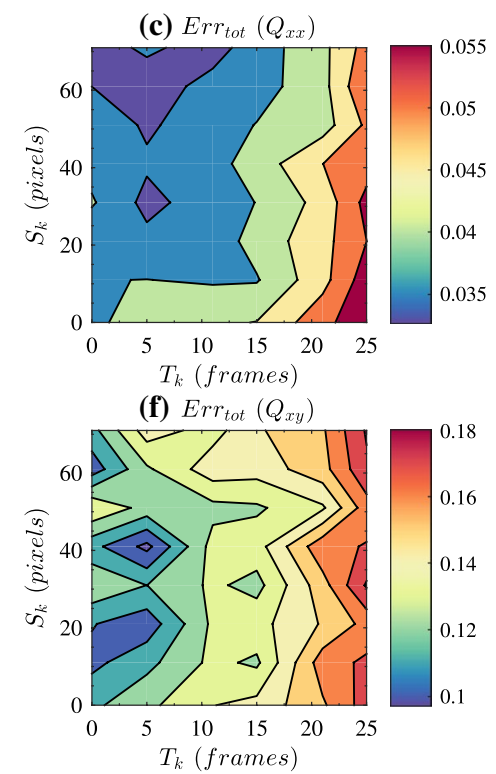

Fig. 9 Predicted identification error as a function of the spatial $\left(S_{k}\right)$ and temporal kernel $\left(T_{k}\right)$ using the special optimised virtual fields method: a systematic error, $\mathbf{b}$ random error and $\mathbf{c}$ total error for $Q_{x x}$. $\mathbf{d}$ Systematic error, $\mathbf{e}$ random error and $\mathbf{f}$ total error for $Q_{x y}$
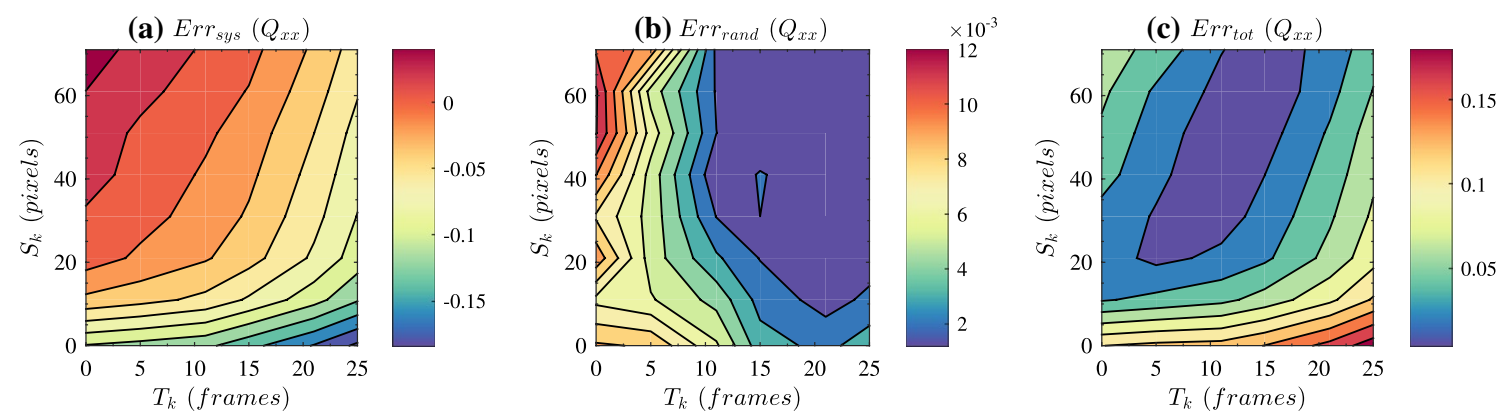

Fig. 10 Predicted identification error as a function of the spatial $\left(S_{k}\right)$ and temporal kernel $\left(T_{k}\right)$ using the stress-gauge equation: a systematic error, b random error and $\mathbf{c}$ total error for $Q_{x x}$ 
the camera resolution (spatial and temporal) is leading to a systematic under prediction of the acceleration compared to the strain, which in turn leads to the identification of a lower stiffness. Further investigation will be required to determine if this bias results from the spatial or temporal resolution of the camera (or both). While there is a systematic bias in the measurement the magnitude is relatively low given the low number of pixels for the simulated camera. The limiting case gives a systematic error of $\sim-5 \%$ for $Q_{x x}$ identified with the optimised virtual fields.

The random error shows the opposite trend to the systematic error with the local minima occurring with significant temporal smoothing and minimal spatial smoothing. In general, the random error maps suggest that the temporal smoothing kernel has a more significant contribution to the random error than the spatial smoothing kernel. This is not surprising as the acceleration is a result of double temporal differentiation which will act to amplify the noise and result in a more significant contribution to the random error. Another trend shown in Figs. 9 and 10 is that the random error tends to be much lower in magnitude than the systematic error. This results from averaging the identified stiffness over a number of frames in the case of the optimised virtual fields or a number of transverse sections in the case of the stress-gauge equation. Another factor contributing to the random error will be the grey level noise of the camera itself. The Shimadzu HPV-X used in this study has excellent image quality with low grey level noise $(0.35 \%$ of the dynamic range) which reduces the random error. Finally, analysis of Fig. 9 shows that the error on the identified $Q_{x y}$ is higher than $Q_{x x}$. This result is expected as the $y$ direction strains and accelerations have a much lower signal to noise ratio than the $x$ direction and the $Q_{x y}$ stiffness parameter is relatively small.
The total error maps show that there are combinations of spatial and temporal smoothing kernels that lead to an optimal compromise between the systematic and random error. Figures 9 and 10 show that the smoothing kernels interact with the optimised virtual fields and stress-gauge equation differently and the local minima for the two methods do not coincide. Therefore the smoothing parameters were selected to minimise the sum of the total error of both methods on the identification of $Q_{x x}$ (referred to as the combined total error). The selected smoothing kernels are as follows: Gaussian over $41 \times 41$ pixels in space and third order Savitsky-Golay over 11 frames in time. In Table 4, the calculated measurement resolutions for the synthetic and experimental images is given for the selected smoothing kernels. Also, the predicted identification errors for this combination of smoothing kernels are given in Table 4. It should be noted that these smoothing parameters do not correspond to the exact minimum combined total error. However, they were selected to minimise the size of the spatial smoothing kernel and reduce edge effects coming from the spatial filter and padding to reconstruct the missing pitch of data. These edge effects are more significant for real grids used in the experiments which can have defects on the edges. The overall difference when compared to the true minimum was $0.15 \%$ on the combined total error for a reduction in the spatial smoothing kernel of 20 pixels.

It is also interesting to note that the predicted total error for $Q_{x x}$ does not vary significantly over a wide range of smoothing kernels. For the optimised virtual fields method the total error is almost always below 5\%, apart from the limit case of the largest temporal smoothing kernels. A similar trend is shown for the stress-gauge equation. Therefore, as long as extreme values of the temporal smoothing kernel are avoided the total error will generally be less than $\sim 5 \%$.
Table 4 Selected smoothing filter kernels with the resulting measurement resolution (for synthetic and experimental images) and predicted identification error

\begin{tabular}{lcll}
\hline Selected filter & \multicolumn{2}{l}{ Type } & Kernel \\
\hline Spatial & \multicolumn{2}{l}{ Gaussian } & $\begin{array}{l}41 \times 41 \text { pixels } \\
11 \text { frames }\end{array}$ \\
Temporal & Savitsky-Golay, third & Experiment & \\
\hline Resolution & 0.91 & 0.76 & Total \\
\hline Raw displacement $(\mu \mathrm{m})$ & 101 & 93 & 3.8 \\
Strain $\left(\mu \mathrm{m} \mathrm{m}^{-1}\right)$ & $2.0 \times 10^{6}$ & $1.6 \times 10^{6}$ & 13 \\
Acceleration $\left(\mathrm{m} \mathrm{s}^{-2}\right)$ & Systematic & Random & 0.50 \\
\hline Error $(\%)$ & -2.4 & 0.70 & 3.7 \\
\hline Virtual fields $Q_{x x}$ & -6.0 & 0.12 & \\
Virtual fields $Q_{x y}$ & -0.26 &
\end{tabular}




\section{Results and Discussion}

\section{Kinematic Fields and Loading Pulse}

Due to the large full-field data set obtained for each specimen the kinematic fields for a single representative specimen will be shown here. For this purpose, specimen 1-WC-F13Co was selected. The full-field displacement maps for this specimen are shown in Fig. 11 at the time point just after the wave has entered the specimen $(t=7.0 \mu \mathrm{s})$ and at a time after the wave has reflected $(t=20.0 \mu \mathrm{s})$. From the displacement both the strain and acceleration fields were numerically derived after smoothing.

The strain fields for specimen 1-WC-F-13Co are shown in Fig. 12. These fields clearly demonstrate the heterogeneous nature of the IBII test. The strain magnitude is quite low with peak local strains on the order of $3 \mathrm{~mm} \mathrm{~m}^{-1}$ for $\epsilon_{x x}$ and approximately half this value for the other strain components. The $\epsilon_{x x}$ strain component clearly shows the compressive pulse entering the specimen in Fig. 12a with the pulse reflecting and becoming tensile as shown in Fig. 12b. The acceleration fields for the same specimen are shown in Fig. 13 with peaks on the order of $8 \times 10^{6} \mathrm{~m} \mathrm{~s}^{-2}$. The strain rate fields for specimen 1-WC-F-13Co are shown in Fig. 14. The compressive strain rate reaches local values on the order of $1000 \mathrm{~s}^{-1}$ and $1500 \mathrm{~s}^{-1}$ in tension. Videos of the kinematic fields for this specimen (1-WC-F-13Co) are provided as supplementary material, videos for all other specimens are provided in the data repository detailed at the end of the manuscript.

Using the average of the acceleration over the field of view the input loading pulse from the projectile can be reconstructed. This is shown in Fig. 15 for specimen
$1-\mathrm{WC}-\mathrm{F}-13 \mathrm{Co}$ in addition to the loading pulse from the image deformation simulations. The experimental pulse peaks at an input load on the order of $200 \mathrm{kN}$ in compression. Comparing the experimental loading pulse to the image deformation pulse shows some significant differences. The image deformation pulse has a much sharper rise time than the experimental pulse. This is expected as the experimental pulse will be shaped by the thin layer of copper tape used to trigger the waveguide as well as the glue used to bond the sample to the waveguide. There will also be some plastic deformation in the projectile leading to a pulse shaping effect and increasing the pulse rise time. The experimental pulse is also much higher in magnitude than the loading pulse predicted by image deformation simulations. It is currently unclear why the experimental pulse is significantly larger in magnitude than the pulse predicted by the simulations. The peak pressure of the impact pulse is normally driven by the impact speed and the material properties of the impactor. The measured impact speed for the pulse shown in Fig. 15 was $50.4 \mathrm{~m} \mathrm{~s}^{-1}$ compared to the simulation which used an impact speed of $50 \mathrm{~m} \mathrm{~s}^{-1}$. Thus, it is possible that a difference in material properties (stiffness or density) is responsible for this discrepancy. Another possibility is that after the projectile exits the barrel it travels a further $250 \mathrm{~mm}$ before impacting the waveguide. Over this distance it is possible that the air escaping from the barrel.

\section{Stiffness Identification}

It is useful to directly compare the stiffness identification with the image deformation simulations presented in "Image Deformation Stiffness Identification" section to experimental data. This comparison can help to verify that the image deformation simulations are accurately representing the
Fig. 11 Displacement fields (unsmoothed) for 1-WC-F-13Co before and after the wave has reflected. a $u_{x}$ field at $t=7.0 \mu \mathrm{s}$ and $\mathbf{b}$ at $t=20.0 \mu$ s. $\mathbf{c} u_{y}$ field at $t=7.0 \mu \mathrm{s}$ and $\mathbf{d}$ at $t=20.0 \mu \mathrm{s}$
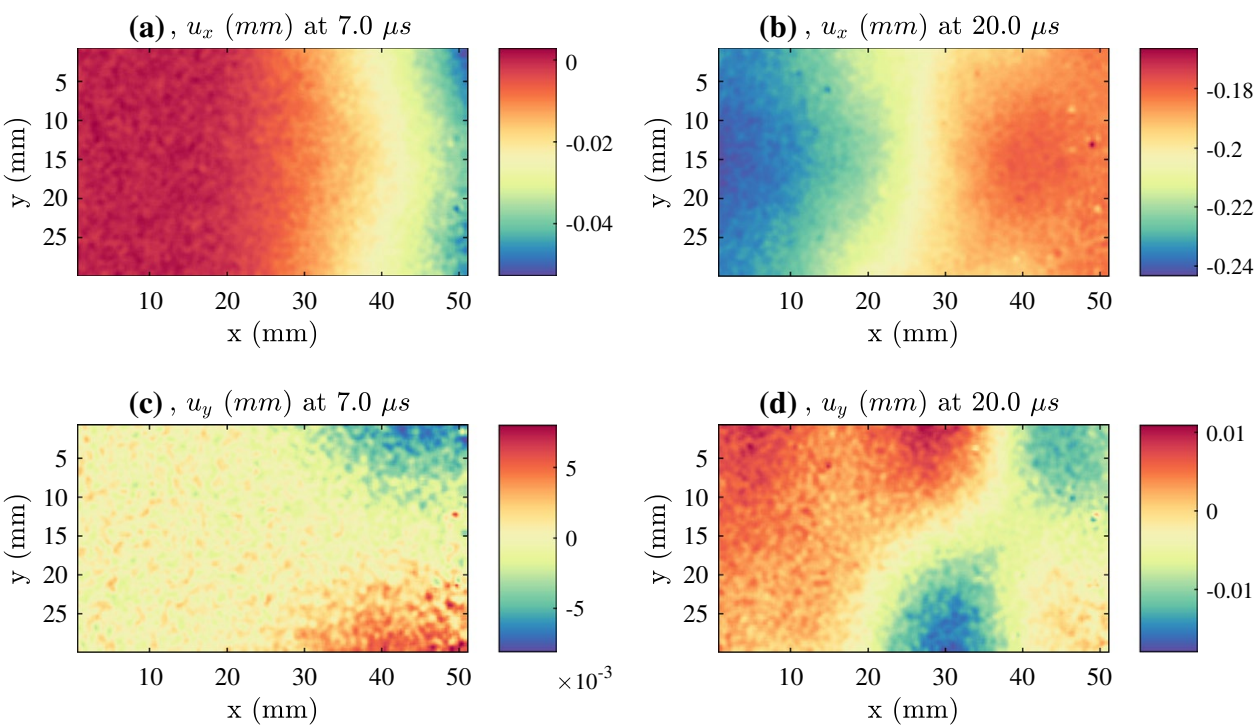
Fig. 12 Strain fields for 1-WCF-13Co before and after the wave has reflected. a $\epsilon_{x x}$ field at $t=7.0 \mu \mathrm{s}$ and $\mathbf{b}$ at $t=20.0 \mu \mathrm{s}$. c $\epsilon_{y y}$ field at $t=7.0 \mu \mathrm{s}$ and $\mathbf{d}$ at $t=20.0 \mu$ s. e $\epsilon_{x y}$ field at $t=7.0 \mu \mathrm{s}$ and $\mathbf{f}$ at $t=20.0 \mu \mathrm{s}$
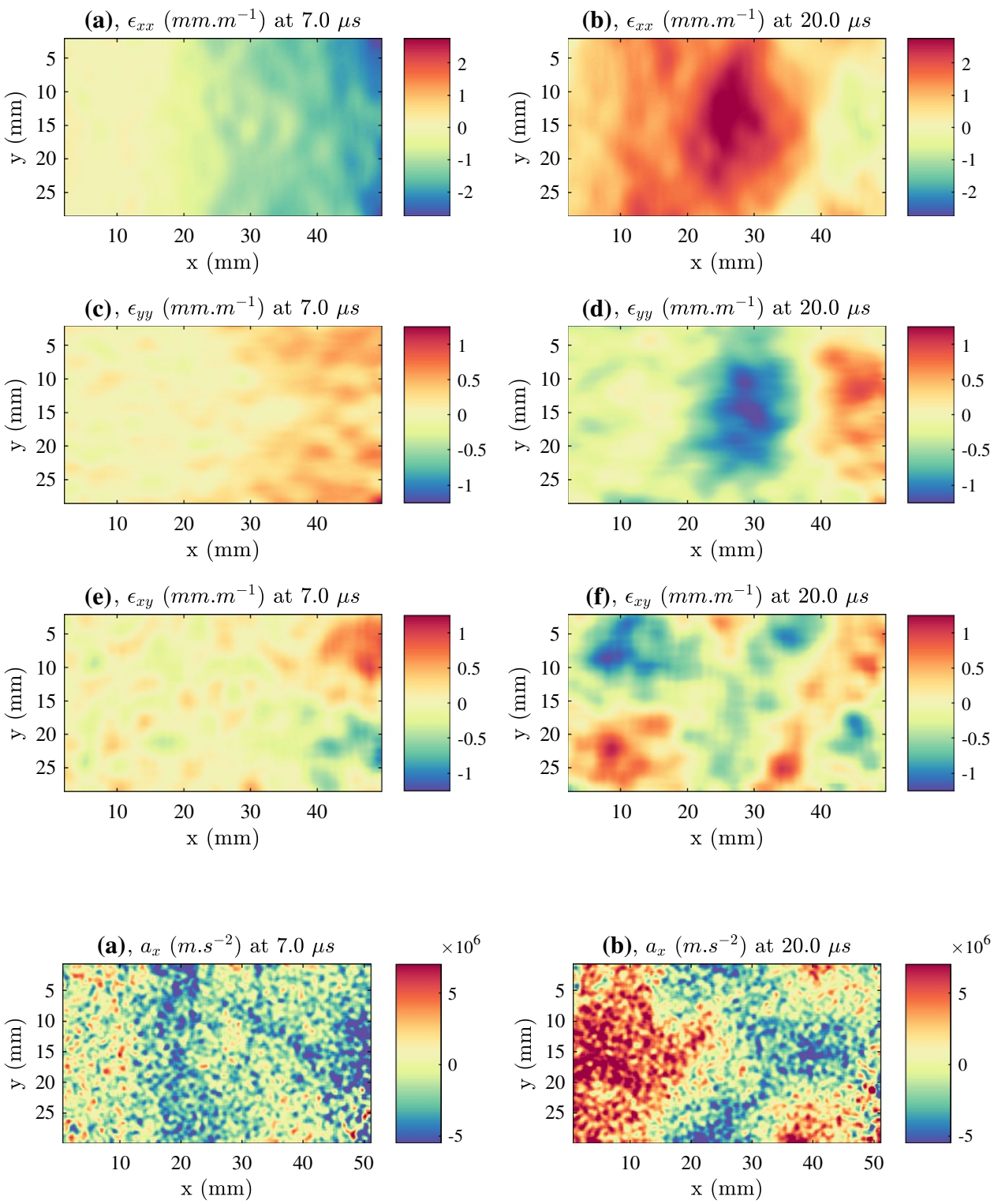

Fig. 13 Acceleration fields for 1-WC-F-13Co before and after the wave has reflected. a $a_{x}$ field at $t=7.0 \mu$ s and b at $t=20.0 \mu$ s. c $a_{y}$ field at $t=7.0 \mu \mathrm{s}$ and $\mathbf{d}$ at $t=20.0 \mu \mathrm{s}$

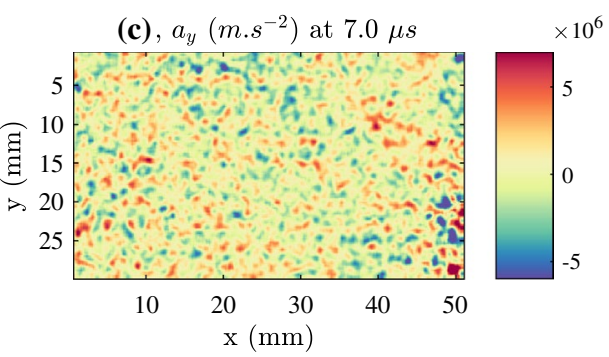

experiments, allowing for appropriate selection of smoothing parameters and error prediction. A comparison of the identified stiffness as a function of time for specimen 1-WC$\mathrm{F}-13 \mathrm{Co}$ and the image deformation simulation is given in Fig. 16. Stress-strain curves for specimen 1-WC-F-13Co are shown in Fig. 17a (compared to Fig. 8a for the image deformation simulation). Finally, the identified stiffness over the specimen length using the stress-gauge equation is shown in Fig. 17b for specimen 1-WC-F-13Co and the image deformation simulation.

The results in Figs. 16 and 17 show that the experiments and image deformation simulations show quite good agreement with a few notable differences. For the optimised virtual fields procedure the experimental data shows slightly 
Fig. 14 Strain rate fields for 1-WC-F-13Co before and after the wave has reflected. a $\epsilon_{x x}^{\cdot}$ field at $t=7.0 \mu$ s and $\mathbf{b}$ at $t=20.0 \mu \mathrm{s}$ (a), $\epsilon_{x x}\left(s^{-1}\right)$ at $7.0 \mu s$

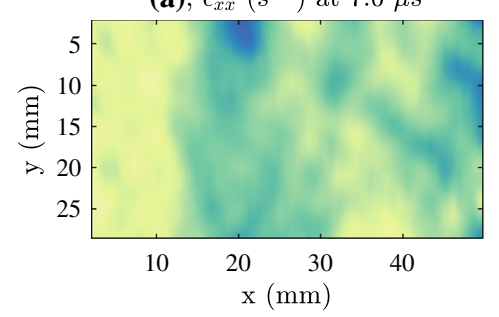

(b), $\epsilon_{x x}^{\cdot}\left(s^{-1}\right)$ at $20.0 \mu s$

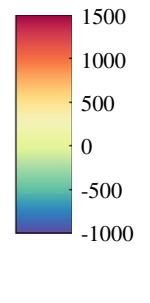

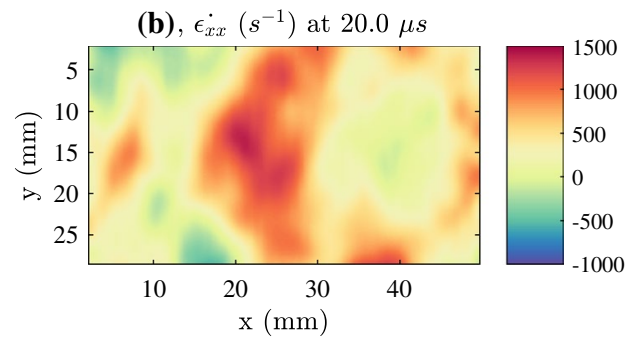

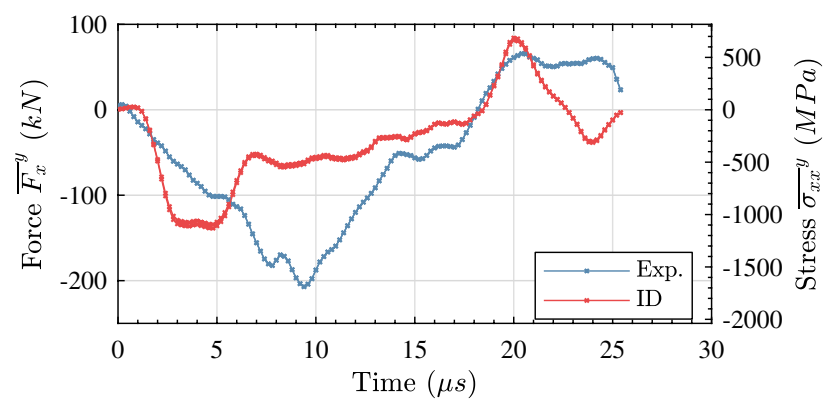

Fig. 15 Input loading pulse calculated from the average acceleration over the field of view for specimen 1-WC-F-13Co (Exp.) and the image deformation simulations (ID.) higher variability than the simulation and greater instability at the point of wave reflection. Also, for this specimen the tensile fracture occurs at $21.4 \mu$ s leading to a drop in stability once the fracture zone becomes large enough to disrupt the identification. Differences between the image deformation simulation and experiments are expected as the image deformation simulation does not account for all experimental errors. The two main sources of error that are not included in the image deformation simulations include grid defects and three-dimensional effects (e.g. non-uniformity of kinematic fields through-thickness). Both of these error sources will be discussed in detail in the limitations section, see "Limitations and FutureWork" section.
Fig. 16 Comparison of the identification using the optimised virtual fields for experiment 1-WC-F-13Co (Ex.) to the image deformation simulation (ID.). a Identification for $Q_{x x}$ and $\mathbf{b} Q_{x y}$

(a)

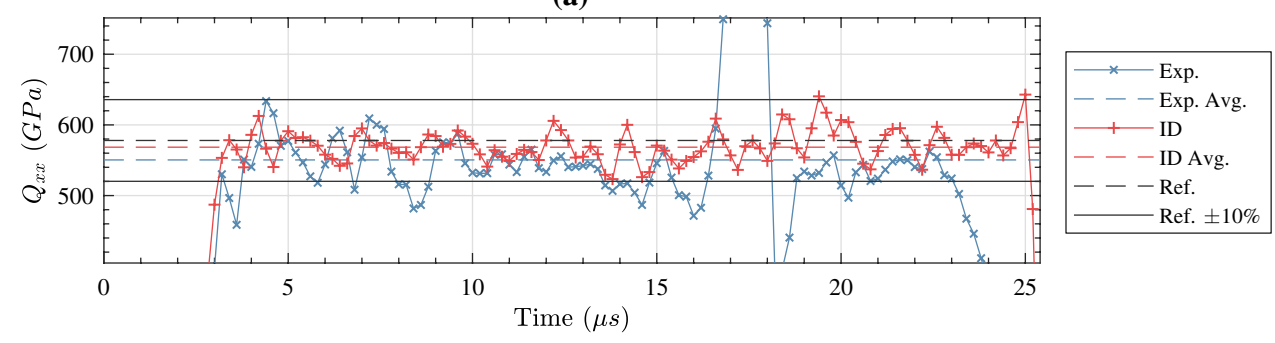

(b)

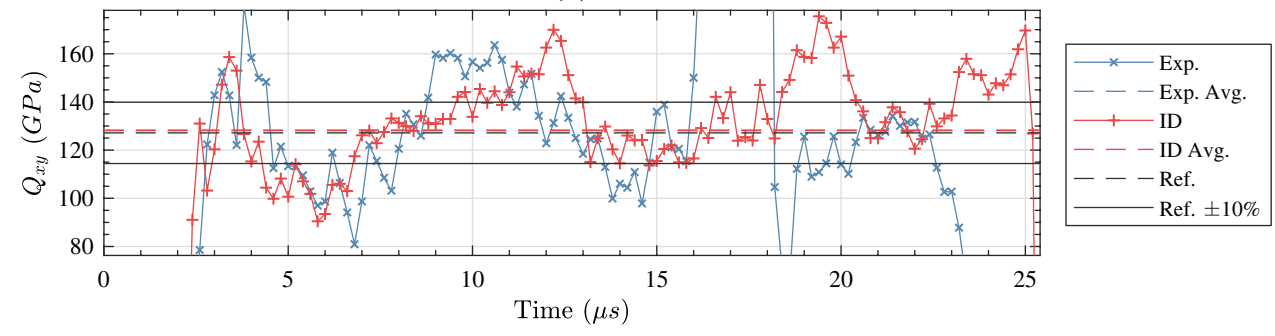

(a)

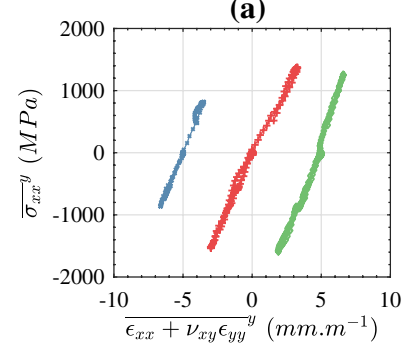

(b)

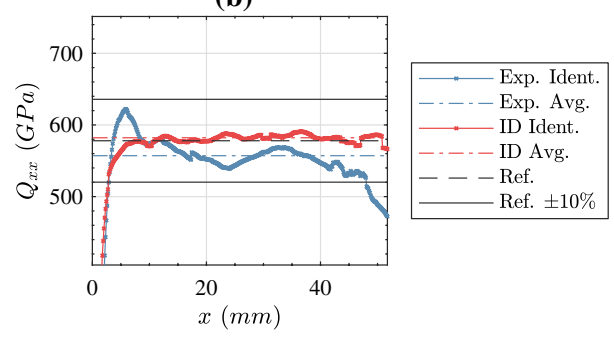


Fig. 18 Identified stiffness using the optimised virtual fields method as a function of time for all specimens tested. a Identification of $Q_{x x}$ and $\mathbf{b}$ identification of $Q_{x y}$. Note that for clarity the time axis has been truncated to only include the time range over which the identification is stable. The reference value provided is the same as used for the image deformation simulations with the reference bounds giving a scale to asses scatter in the data (a)
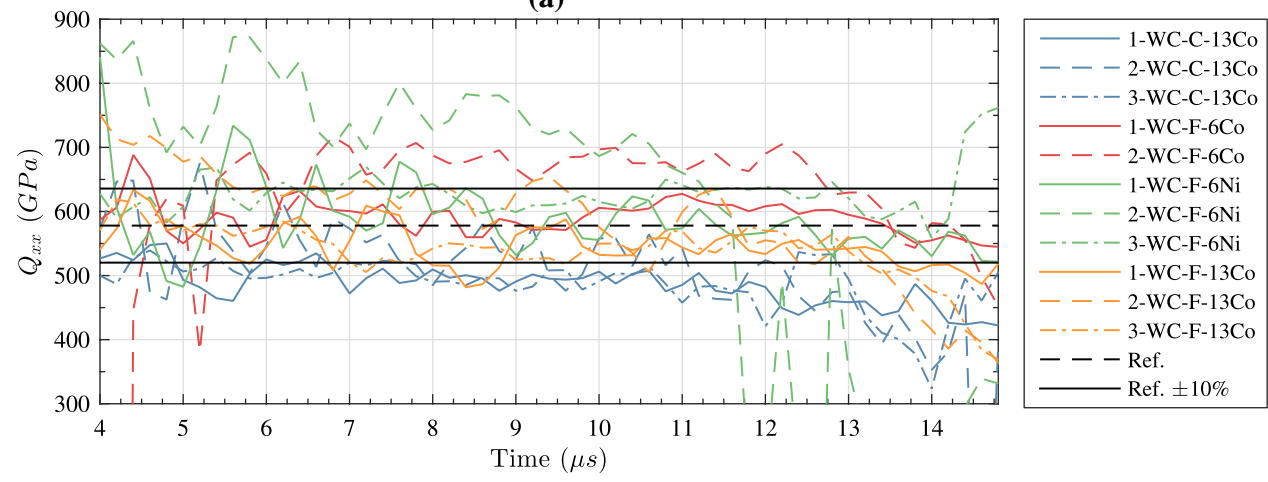

(b)

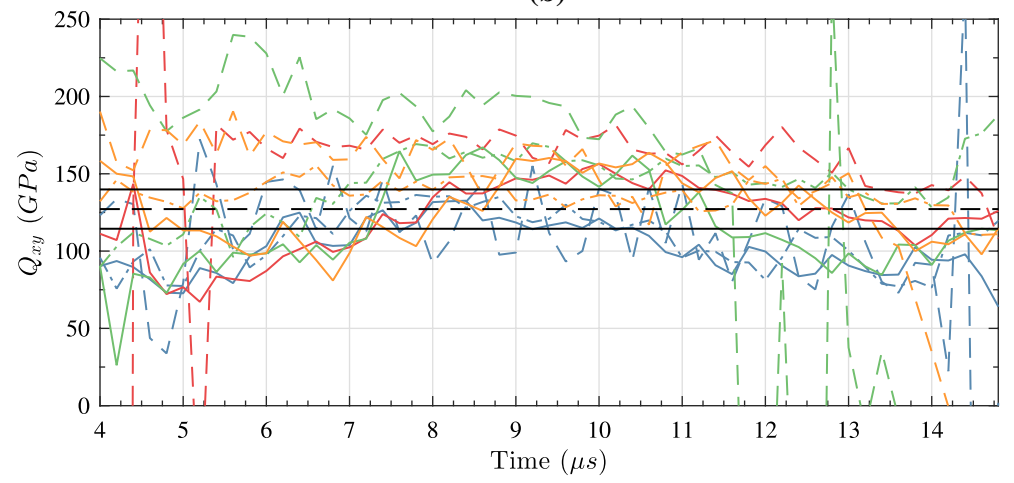

Table 5 Identified isotropic stiffness components using the optimised virtual fields method (VF) and stress-gauge equation (SG)

\begin{tabular}{llllllll}
\hline Specimen & $Q_{x x}(V F)(\mathrm{GPa})$ & $Q_{x y}(\mathrm{VF})(\mathrm{GPa})$ & $E(V F)(\mathrm{GPa})$ & $v(V F)$ & $Q_{x x}(\mathrm{SG})(\mathrm{GPa})$ & $E(S G)(\mathrm{GPa})$ & ${\overline{\epsilon_{x x}^{-}}}^{-}(\mathrm{min})\left(s^{-1}\right)$ \\
\hline 1-WC-C-13Co & 496 & 114 & 470 & 0.23 & 545 & 516 & -1240 \\
2-WC-C-13Co & 538 & 112 & 515 & 0.21 & 532 & 509 & -1120 \\
3-WC-C-13Co & 498 & 119 & 470 & 0.24 & 543 & 512 & -737 \\
Average & 512 & 115 & 485 & 0.23 & 539 & 512 & -1030 \\
1-WC-F-6Co & 599 & 136 & 568 & 0.23 & 642 & 511 & -1170 \\
2-WC-F-6Co & 675 & 170 & 632 & 0.25 & 631 & 592 & -663 \\
Average & 637 & 153 & 600 & 0.24 & 637 & 500 & -916 \\
1-WC-F-6Ni & 592 & 131 & 563 & 0.22 & 597 & 568 & -1250 \\
2-WC-F-6Ni & 729 & 192 & 678 & 0.26 & 610 & 567 & -1360 \\
3-WC-F-6Ni & 628 & 151 & 592 & 0.24 & 607 & 571 & -1650 \\
Average & 649 & 158 & 611 & 0.24 & 604 & 569 & -1420 \\
1-WC-F-13Co & 550 & 128 & 521 & 0.23 & 557 & 527 & -140 \\
2-WC-F-13Co & 628 & 165 & 585 & 0.26 & 554 & 516 & -1200 \\
3-WC-F-13Co & 549 & 137 & 515 & 0.25 & 547 & 513 & \\
Average & 576 & 143 & 540 & 0.25 & 553 & 519 & \\
\hline
\end{tabular}

The maximum width averaged compressive strain rate is also provided

The identification of the isotropic stiffness components using the optimised virtual fields is shown in Fig. 18 for all specimens over the time period for which the identification is stable. The identification is quite consistent between samples and compares well to the image deformation simulations. The average identified stiffness components for each sample are summarised in Table 5. Unfortunately, it was not possible to identify the stiffness for specimen 3-WC-F-6Co due to difficulties bonding a grid to the sample. The identified stiffness $Q_{x x}$ using the stress-gauge equation is shown in Fig. 19 for all specimens with the average stiffness identified for each sampled being summarised in Table 5. As predicted 


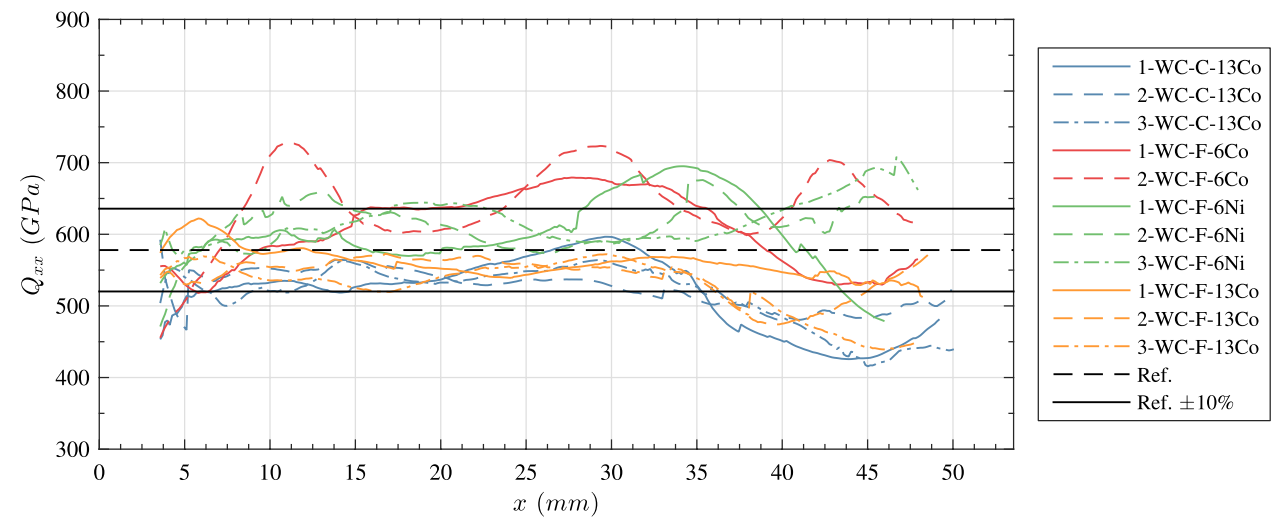

Fig. 19 Identification of $Q_{x x}$ using the stress gauge equation for all specimens tested. Note that data within one grid pitch plus half a smoothing kernel ( 25 pixels) has been removed as this is corrupted by edge effects. For comparison purposes the $y$ axis has been set to the same limits as Fig. 18a. The reference value provided is the same as used for the image deformation simulations with the reference bounds giving a scale to asses scatter in the data

et al. [16] tested a wide variety of tungsten carbide cermets in quasi-static compression measuring both the elastic modulus and Poisson's ratio using strain gauges. From the data in Getting et al. the elastic modulus of tungsten carbide cermets with $6 \%$ cobalt binder lies between 625 and $638 \mathrm{GPa}$ while cermets with $12 \%$ cobalt binder have a modulus between 540 and $564 \mathrm{GPa}$. The Poisson's ratio for all grades tested in [16] was between 0.19 and 0.22 . These results compare quite well to results in Table 5 with the modulus values being lower than those of [16] ( $\sim 6 \%$ for the $6 \%$ binder and $\sim 8 \%$ comparing with the $12 \%$ binder from [16] to the $13 \%$ binder used in this study). The results of this study find a Poisson's ratio that is higher than [16] by $\sim 20 \%$. It is possible that this difference comes from differences in material constituents and manufacturing procedures as the cermets tested in [16] come from other manufacturers. However, it should also be noted here that some of the difference can be attributed to the experimental error as predicted by the image deformation simulations and the experimental errors in [16].

Overall, the results for the stiffness identification are extremely promising, given that the tungsten carbide cermets tested here are stiff compared to many engineering materials and exhibit quite low strains. This suggests that the IBII test is a useful tool for investigating the stiffness of ceramic composites at high strain rates. It is also encouraging that the stiffness identification for the tungsten carbide cermets tested here agree closely with literature values for the quasi-static stiffness. This confirms that there is no strain rate dependence for the elastic properties of tungsten carbide cermets at strain rates up to $1000 \mathrm{~s}^{-1}$.

\section{Strength Identification}

The tensile strength was determined for all specimens using the procedure described in [10]. This procedure will be in [21] only gives a lower bound on the true value. Getting 


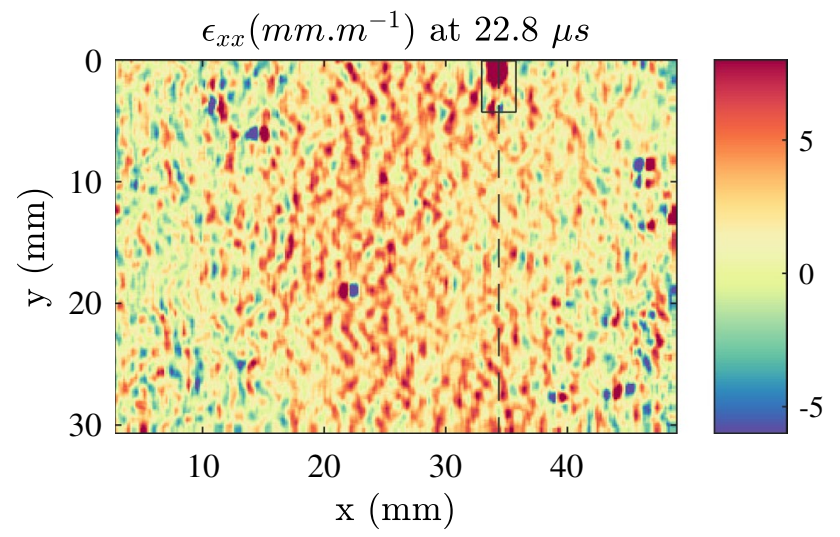

Fig. 20 Raw strain map showing the fracture location and virtual gauge area for specimen 1-WC-F-13Co

briefly outlined here using specimen 1-WC-F-13Co as an example. The first stage of the strength identification is to identify the first crack location from the raw unsmoothed strain maps. The crack location for specimen 1-WC-F-13Co is shown in Fig. 20. A virtual gauge area is then used to identify the strength at the crack location using the linear stress gauge equation. Here the virtual gauge had a window of $20 \times 30$ pixels $[x, y]$, this area is illustrated in Figs. 20 and $21 \mathrm{a}$ and $\mathrm{b}$. The strength is taken as the maximum stress averaged over the gauge area using the stress field from the linear stress-gauge equation, denoted as ${\overline{\sigma_{x x}, u l t}}^{A}(L S G)$. The stress field from the linear stress-gauge equation is shown in Fig. 21a at the time in which the maximum stress is reached in the virtual gauge area. This shows reasonable agreement with the stress field reconstructed from the strain using the identified constitutive parameters as shown in Fig. 21b.
Two measures of stress can be obtained at the virtual gauge location, the first is from the linear stress-gauge equation and the second is calculated from the strains using the stiffness parameters identified previously. When the material fails the strains become non-physical and these two measures of stress will diverge. This is illustrated in $21 \mathrm{c}$. The stress over the gauge area calculated from the linear stress-gauge can also be compared to the axial average stress at the fracture location $\left({\overline{\sigma_{x x}}}^{y}\right)$. This gives an indication of how much the moment term (second term in Eq. 11) is contributing to the strength or how 'uni-axial' the test is. It is also possible to construct a local stress strain curve over the gauge area as shown in Fig. 21d. For specimen 1-WC-F-13Co shown in Fig. 21 averaging over the small gauge area leads to a local stress-strain curve which is relatively noisy compared to those shown in Fig. 17a. This is a result of averaging the strains over a small window and the contribution of the moment term in the linear stress-gauge equation. This term includes spatial averages of $a_{y}$ which has a low signal to noise ratio. This is clearly shown in Fig. $21 \mathrm{c}$ when comparing the different stress measures; the ${\overline{\sigma_{x x}, u l t}}^{A}(L S G)$ term has the largest variability over time. Comparison of the fields in Fig. 21a and b shows that fracture does not occur at the location in the sample experiencing the highest strain and tends to occurs at the edge of the sample. As mentioned previously, cermets like tungsten carbide are defect sensitive so the specimen will not necessarily fail at the location experiencing the highest stress.

It was possible to use this methodology to identify the strength for all tested samples except 3-WC-C-13Co which did not fail during the portion of the test that was imaged. Therefore the strength value reported for this specimen is the maximum LSG stress value through the recording period. The identified tensile strength for all specimens is
Fig. 21 Strength diagnostics for 1-WC-F-13Co. a Stress field from the linear stress-gauge equation (LSG) (Eq. 11). b Stress field calculated from the measured strains using the identified constitutive parameters. c Comparison of stress measures as a function of time over the virtual gauge region. $\mathbf{d}$ Local stress-strain curve over the virtual gauge area
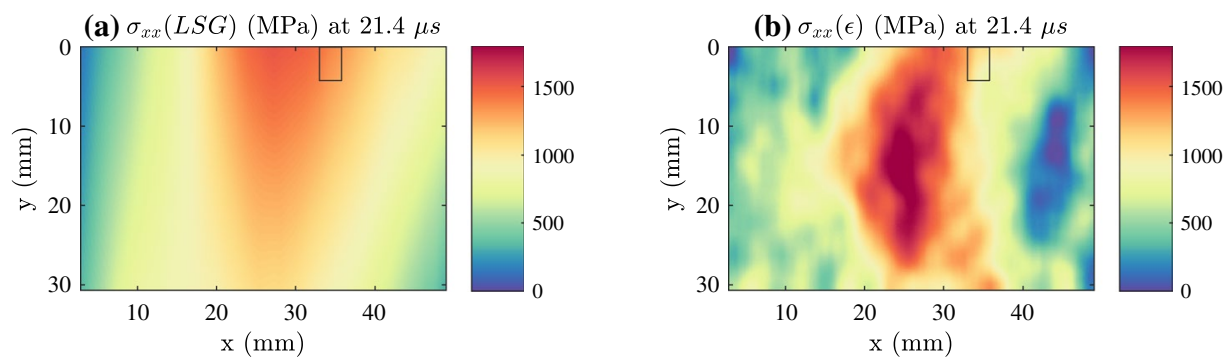

(c)
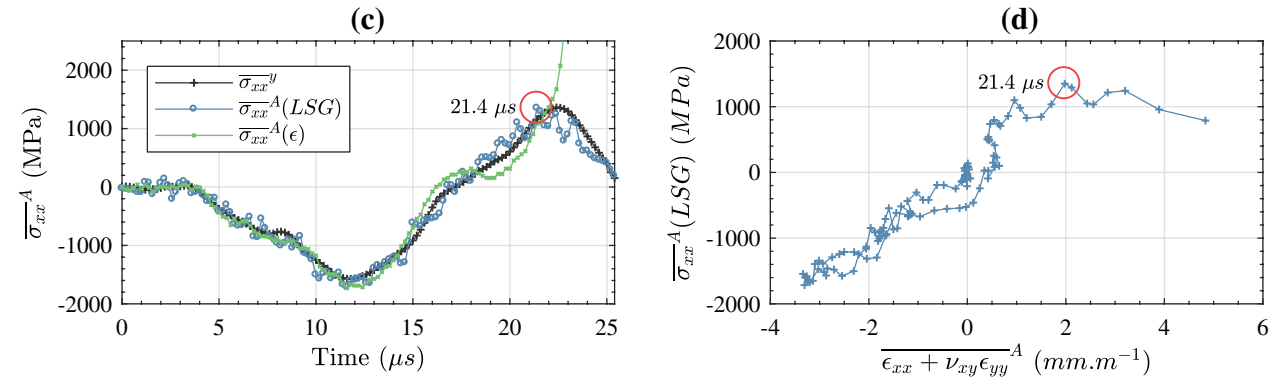
Table 6 Identified strength for all tested samples using the linear stress-gauge equation $\left({\overline{\sigma_{x x}, u l t}}^{A},(L S G)\right)$ with the standard stress-gauge provided for comparison $\left(\frac{\sigma_{x x}, u l t}{y}(S G)\right)$

\begin{tabular}{|c|c|c|c|}
\hline Specimen & ${\overline{\sigma_{x x}}}_{, \text {ult }}^{y}(S G)(\mathrm{MPa})$ & ${\overline{\sigma_{x x}, u l t}}^{A}(L S G)(\mathrm{MPa})$ & ${\overline{\epsilon_{x x}}}^{A} \max \left(s^{-1}\right)$ \\
\hline $1-W C-C-13 C o$ & 1250 & 1480 & 810 \\
\hline 2-WC-C-13Co & 1310 & 1820 & 990 \\
\hline 3-WC-C-13Co & $1060^{*}$ & $1340^{*}$ & 920 \\
\hline Average & 1210 & 1550 & 910 \\
\hline $\begin{array}{l}\text { QS. } 0.5 \sigma_{T R S} \\
(\mathrm{MPa})\end{array}$ & 1740 & & \\
\hline 1-WC-F-6Co & 1250 & 1240 & 1000 \\
\hline 2-WC-F-6Co & 872 & 1550 & 800 \\
\hline Average & 1060 & 1390 & 900 \\
\hline $\begin{array}{l}\text { QS. } 0.5 \sigma_{T R S} \\
\text { (MPa) }\end{array}$ & 1170 & & \\
\hline 1-WC-F-6Ni & 666 & 820 & 1400 \\
\hline 2-WC-F-6Ni & 788 & 1290 & 980 \\
\hline 3-WC-F-6Ni & 940 & 1070 & 1700 \\
\hline Average & 798 & 1060 & 1400 \\
\hline $\begin{array}{l}\text { QS. } 0.5 \sigma_{T R S} \\
(\mathrm{MPa})\end{array}$ & 1330 & & \\
\hline 1-WC-F-13Co & 1370 & 1350 & 1200 \\
\hline 2-WC-F-13Co & 1150 & 1460 & 930 \\
\hline 3-WC-F-13Co & 918 & 1245 & 920 \\
\hline Average & 1140 & 1350 & 1000 \\
\hline $\begin{array}{l}\text { QS. } 0.5 \sigma_{T R S} \\
(\mathrm{MPa})\end{array}$ & 1590 & & \\
\hline
\end{tabular}

*Did not fail during recording, maximum values reported

The quasi-static (QS.) reference strength is estimated as half the transverse rupture strength from [21] summarised in Table 6. A comparison of the stress measures over time for a specimen from each group is shown in Fig. 22. These samples were selected to illustrate the different behaviours observed across all samples. In general, the linear stress-gauge provides the best agreement with the measured strain over the virtual gauge area, for example 2-WC-F-6Co and 2-WC-F-13Co in Fig. 22b and d respectively. However, for some specimens there is no significant difference between the standard stress gauge and the linear stress-gauge, see 1-WC-F-13Co in Fig. 21c and 3-WC-F-6Ni in Fig. 22c. Comparison of standard and linear stress gauge gives an indication of the in-plane tilt of the sample during the test. The larger the moment term of the linear stressgauge equation the larger the tilt.

The most interesting behaviour is exhibited by specimen 1-WC-C-13Co shown in 22a. For this specimen the stress measures all follow closely until $17 \mu$ s into the test when they diverge while still in compression. A similar behaviour was shown by specimen 1-WC-F-6Co. It is possible that this is a result of three-dimensional effects. However, one would expect this to manifest immediately following the wave reflection from the free edge which is not the case here. The ' $S$ ' shape of the stress calculated from the strain is similar to that shown by $1-\mathrm{WC}-\mathrm{F}-13 \mathrm{Co}$ (Fig. 21c). It is interesting to note here that a similar ' $\mathrm{S}$ ' shaped strain profile is predicted by the image deformation simulations but given the difference in input loading pulse (see Fig. 15) care needs to be taken in making this comparison. The fact that something similar appears in the image deformation simulations suggests that the cause of this divergence may be the error generated by reconstructing data at the free edge by extrapolation. If this is the case then future improvements in camera technology with larger pixel array sizes will significantly reduce this error. However, further experiments
Fig. 22 Stress measures at the fracture location for one specimen from each group. These specimens were selected to represent the different behaviours observed across all tested specimens
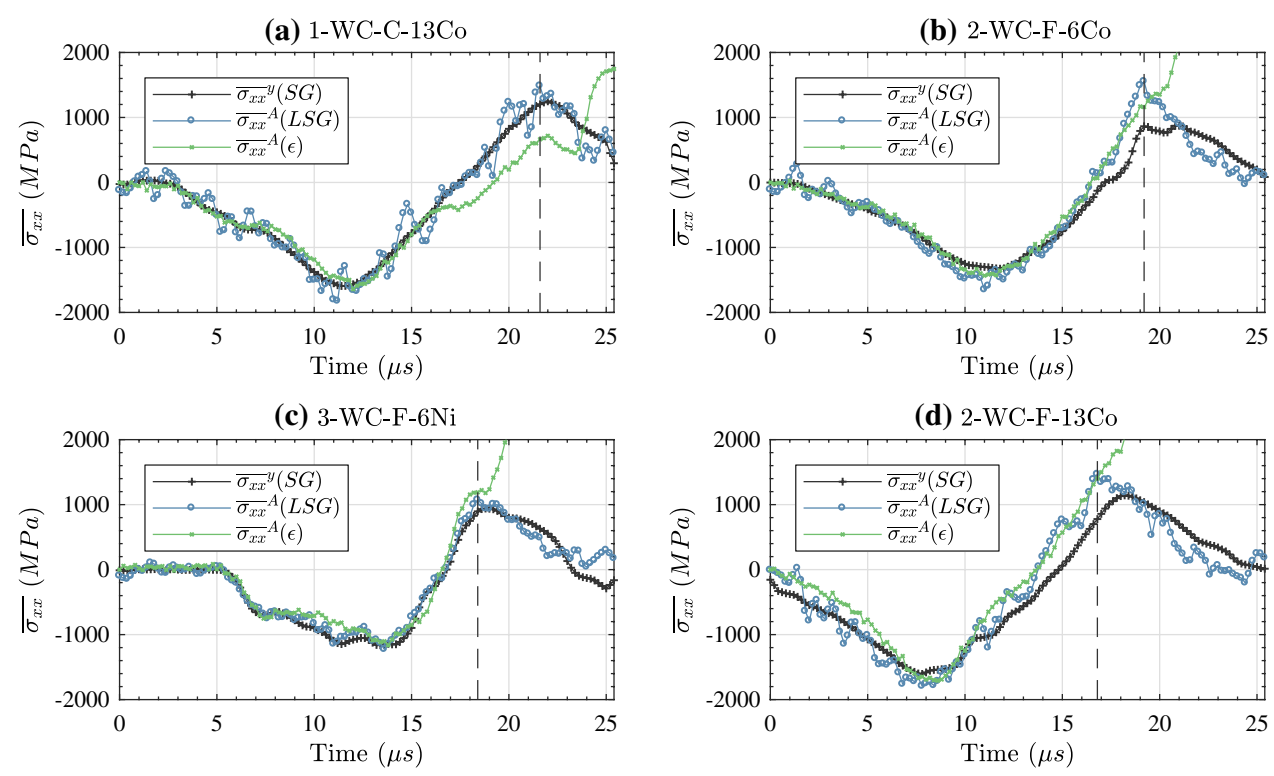
are needed to verify that three-dimensional effects are not responsible by using a back-to-back imaging procedure. This is discussed in further detail in the limitations section (see "Limitations and FutureWork" section ).

As data for the quasi-static tensile strength of tungsten carbide is not available it is difficult to comment on any strain rate sensitivity of the measured properties. Furthermore, it is well known that ceramics have considerable variability in measured strength which is correlated with the sample volume $[17,20]$. While stressed volume effects are well characterised for the case of quasi-static loading this becomes more complicated for the case of dynamic loading because the stress distribution is time dependent (i.e. there is stress wave propagation). Leaving the problem of dynamic stressed volume effects aside, it is possible to have an estimate of the quasi-static tensile strength by using the transverse rupture strength taken from a bending test as described in "Model Configuration and Design Sweep Criteria" section and [17]. Based on the three-point bending failure load reported in [21] the tensile strength can be estimated for the given grades of tungsten carbide as half the transverse rupture strength, $0.5 \sigma_{T R S}$. These results are provided for comparison in Table 6. Comparing to the quasi-static strength estimations the dynamic experiments in this study mostly have a lower strength by 200-300 MPa apart from the WCF-6Co samples which show the opposite trend. Considering that the quasi-static strength estimates are not significantly outside the range of strength values reported for each group it is unlikely that there is any strain rate dependence for the tensile strength up to $\sim 1000 \mathrm{~s}^{-1}$. It should be noted that the sample sizes tested here are not meant to be large enough to perform statistical tests. However, this study clearly demonstrates that this methodology is a viable method for obtaining high strain rate strength data for brittle materials and could be used for larger investigations in the future.

At this point it is worth considering that the image deformation sweep used to select the smoothing parameters optimised the error on the identified stiffness components not the strength identification. It would be desirable to develop an image deformation procedure to select optimal smoothing kernels for the strength identification and to estimate the associated error in the identified strength. This will be undertaken as future work and is discussed further in the following section.

\section{Limitations and Future Work}

This study has shown that the IBII test is an excellent tool for investigating the high strain rate properties of brittle materials, using tungsten carbide cermets as an example. However, it is useful to discuss the limitations that have arisen when discussing the results as these give an indication of how the method can be improved in the future. The two main limitations of the method include grid defects and threedimensional effects which are discussed further below.

\section{Grid Defects}

This refers to small areas of missing grid that occur due to bubbles in the glue used to bond the grid to the sample. Grid defects can also manifest as small areas of grid that chip off the edge of the sample during the grid transfer procedure. These areas of missing grid have an effect on the variability of the identified properties as they vary from sample to sample. The errors associated with grid defects could be quantified using the image deformation procedures detailed in this work. Instead of using an ideal synthetic grid, a static image of an experimental specimen could be deformed by sub-pixel interpolation (as described in [28]). This procedure could be used to predict the error for each individual specimen. However, this is beyond the scope of the present work and may not be necessary as a new method of grid deposition has been developed which drastically reduces the occurrence of grid defects. This new method allows grids to be printed directly onto samples using a flat bed UV ink jet printer with pitches down to $0.33 \mathrm{~mm}$. This has drastically reduced the problem of grid defects (especially for defects on the edges of the sample) and will be implemented where possible in future experiments.

\section{Three-Dimensional Effects}

There are two key assumptions associated with the data processing used in these experiments. The first is that the specimen is in a state of plane stress and the second is that the kinematic fields are uniform through the thickness of the sample. The combination of these assumptions is equivalent to assuming that there is no through-thickness dispersion and that the test is only two dimensional. In practice, significant misalignment at impact or geometrical imperfections of the sample could potentially lead to through-thickness dispersion. Obviously, it would be desirable to quantify if these assumptions hold and if they do not, what the resulting error on the stiffness and strength identification is. Here the ratio of thickness to the smallest in plane dimension is not unfavourable (height of $30 \mathrm{~mm}$ and thickness of $4 \mathrm{~mm}$ ) so it is unlikely that the plane stress assumption has a significant effect. However, this could be investigated using the image deformation simulation procedure described in "Image Deformation Simulations" section by using a three-dimensional finite element model where the thickness of the sample is progressively increased. This model could also be used to investigate the effects of geometrical imperfections such as non-square edges of the specimen. This will be undertaken further as future work. To verify the uniformity of the 
kinematic fields the experiment can be imaged in a back-toback configuration using two cameras. The kinematic fields on the front and back of the sample can then be matched to see if there is any through thickness non-uniformity. These experiments will also be conducted as future work.

With these limitations in mind it is useful to consider possible future work for developing the IBII method further and as well as other applications of the IBII test for obtaining high strain rate properties of brittle materials.

\section{Error Analysis for Strength Identification}

The image deformation procedure presented in this study focused on selecting smoothing parameters to minimise the error on the identified stiffness. It is likely that these are not the optimal parameters for identifying the strength of the material which is mostly dependent on the measured acceleration and not a combination of acceleration and strain. Furthermore, it was found that a significant contribution to the noise in the stress measured at the fracture location came from the moment term of the linear stress-gauge equation as a result of the $a_{y}$ acceleration. It will be useful to investigate using different smoothing kernels on the $x$ and $y$ components of the kinematic fields for strength identification purposes. Therefore, in the future an image deformation procedure will be developed for minimising the error on the identified strength. This will include using an explicit dynamics cohesive zone model to replicate crack growth. The displacement fields from this model can then be used to synthetically deform images, select optimal smoothing parameters and quantify the errors associated with the strength identification procedure.

Application to Other Brittle Materials This study has demonstrated the potential of the IBII test for brittle materials with high stiffness and strength. There are several other materials that exhibit low strains to failure for which the high strain rate behaviour is of interest. This includes, sodalime glass, concrete, graphite, sapphire, boron carbide and silicon carbide. One of the most challenging cases will be testing armour ceramics such as boron and silicon carbide. These materials have bulk wave speeds on the order of $10 \mathrm{~km} \mathrm{~s}^{-1}$ and will present a particular challenge to the temporal resolution of the camera which is currently limited to $5 \mathrm{Mfps}$ at full resolution. Image deformation simulations will be extremely useful for designing the IBII test for these materials to understand and minimise identification errors.

\section{Conclusion}

The IBII test was successfully used to identify the high strain rate properties of various grades of tungsten carbide at strain rates on the order of $1000 \mathrm{~s}^{-1}$. This study used the IBII methodology to produce the first high strain rate data for the stiffness and tensile strength of tungsten carbide cermets. The results of this study are summarised as follows:

1. Image deformation simulations are a vital tool for developing and assessing test methods based on full-field measurements. These simulations allow for the optimal selection of processing parameters and the estimation of the associated measurement errors.

2. The IBII test is extremely well suited for testing brittle materials at high strain rates as shown by the successful identification of the stiffness and strength of a series of tungsten carbide cermets at strain rates on the order of $1000 \mathrm{~s}^{-1}$. This methodology does not require the sample to be gripped and does not require significant modification to test high stiffness and high strength materials.

3. The identified stiffness components of all grades of tungsten carbide tested showed no significant strain rate dependence. Furthermore, the trends in the identified elastic modulus for different grades correlated well with quasi-static results (i.e. higher binder content leads to lower modulus).

4. The identified tensile strength for all tested cermets showed negligible strain rate dependence when compared to estimates of the quasi-static tensile strength taken form transverse rupture tests.

Overall, this study demonstrates the potential of full-field measurements to provide high strain rate test data for brittle materials that is not currently available with other methodologies. In the future, ultra-high speed camera technology will improve leading to larger pixel arrays, higher frame rates and longer recording times. This will decrease measurement errors and allow for more complex tests to be performed. The authors hope that the IBII test method will become a standard test for obtaining high strain rate properties for brittle materials in the future.

Acknowledgements The authors want to thank Dr Leslie Lamberson from Drexel University for providing the samples and for useful discussions about the material. Dr Lloyd Fletcher and Prof. Fabrice Pierron acknowledge support from EPSRC through Grant EP/L026910/1.

Data Provision All data supporting this study are openly available from the University of Southampton repository at https://doi.org/10.5258/ SOTON/D0607.

Open Access This article is distributed under the terms of the Creative Commons Attribution 4.0 International License (http://creat ivecommons.org/licenses/by/4.0/), which permits unrestricted use, 
distribution, and reproduction in any medium, provided you give appropriate credit to the original author(s) and the source, provide a link to the Creative Commons license, and indicate if changes were made.

\section{Appendix}

\section{Derivation of the Linear Stress-Gauge Equation}

Here rigid body virtual fields will be used to illustrate the derivation of Eq. 11 as described in [10]. The case under consideration is a thin plate sample impacted on one edge as described in Fig. 1. The first virtual field describes a rigid translation in the $x$ direction:

$\left\{\begin{array}{l}u_{x}^{*}=1 \\ u_{y}^{*}=0\end{array}\left\{\begin{array}{l}\epsilon_{x x}^{*}=0 \\ \epsilon_{y y}^{*}=0 \\ \epsilon_{x y}^{*}=0\end{array}\right.\right.$

Starting with the principal of virtual work (Eq. 1), three key assumptions are used to simplify the analysis: (1) the density, thickness and material properties of the specimen do not vary in space; (2) the kinematic fields are uniform through the thickness; and 3) the specimen is in a state of plane stress. Substituting the rigid body virtual field in Eq. (16) into the principal of virtual work gives:

$\int_{l} T_{x} d l=\rho \int_{S} a_{x} d S$

where $S$ is the surface of the specimen and $l$ is the boundary of the specimen. As the traction vector $\boldsymbol{T}=\boldsymbol{\sigma} \cdot \boldsymbol{n}, T_{x}$ is given by the stress component $\sigma_{x x}$. Equation (17) now becomes:

$\int_{-h_{s} / 2}^{h_{s} / 2} \sigma_{x x} d y=\rho \int_{-h_{s} / 2}^{h_{s} / 2} \int_{0}^{x} a_{x} d x d y$

Using overline notation to denote spatial averages (as described at the beginning of "Virtual Fields for Stiffness Identification" section) Eq. (18) simplifies to the stressgauge equation (Eq. 9).

Next, consider the virtual field that describes a rigid body translation in the $y$ direction:

$\left\{\begin{array}{l}u_{x}^{*}=0 \\ u_{y}^{*}=1\end{array}\left\{\begin{array}{l}\epsilon_{x x}^{*}=0 \\ \epsilon_{y y}^{*}=0 \\ \epsilon_{x y}^{*}=0\end{array}\right.\right.$

Applying the same assumptions as described for the first rigid body virtual field the principle of virtual work becomes: $\int_{-h_{s} / 2}^{h_{s} / 2} \sigma_{x y} d y=\rho \int_{-h_{s} / 2}^{h_{S} / 2} \int_{0}^{x} a_{y} d x d y$

Using the overline notation in similar manner yields:

${\overline{\sigma_{x y}}}^{y}=\rho x \bar{a}_{y}^{S}$

where ${\overline{\sigma_{x y}}}^{y}$ is the average shear stress in the cross section at the location $x$ and $\bar{a}_{y}^{S}$ is the surface average of the acceleration from the free edge to $x$.

The final virtual field to be considered is a rigid body rotation about the origin:

$\left\{\begin{array}{l}u_{x}^{*}=y \\ u_{y}^{*}=-x\end{array}\left\{\begin{array}{l}\epsilon_{x x}^{*}=0 \\ \epsilon_{y y}^{*}=0 \\ \epsilon_{x y}^{*}=0\end{array}\right.\right.$

Using the same assumptions as for the previous rigid body virtual fields, the principle of virtual work becomes:

$$
\begin{aligned}
& \int_{-h_{s} / 2}^{h_{s} / 2} \sigma_{x x} y d y-x \int_{-h_{s} / 2}^{h_{s} / 2} \sigma_{x y} d y \\
& \quad=\rho \int_{-h_{s} / 2}^{h_{s} / 2} \int_{0}^{x}\left(a_{x} y-a_{y} x\right) d x d y
\end{aligned}
$$

Applying the overline notation yields:

${\overline{\sigma_{x x} y}}^{y}-x{\overline{\sigma_{x y}}}^{y}=\rho x\left({\overline{a_{x} y}}^{S}-{\overline{a_{y} x}}^{S}\right)$

Now, Eq. (21) can be substituted into Eq. (24) to give:

${\overline{\sigma_{x x} y}}^{y}=\rho x\left(x{\overline{a_{y}}}^{S}+{\overline{a_{x} y}}^{S}-{\overline{a_{y} x}}^{S}\right)$

where ${\overline{\sigma_{x x}}}^{y}$ is the average of the first moment of the axial stress over the cross section at $x$ and the acceleration terms in Eq. (25) are spatial averages over the specimen surface up to the transverse slice $x$.

Equations ( 9, 21 and 25) can now be combined to give a linear approximation of the axial stress distribution. Let the linear approximation of the $\sigma_{x x}$ stress along $y$ be denoted $\sigma_{x x}(L S G)$ (LSG for 'Linear Stress-Gauge'). $\sigma_{x x}(L S G)$ can be expressed as follows:

$\sigma_{x x}(L S G)=\sigma_{x x}^{a}+\frac{y}{h_{s}} \sigma_{x x}^{b}$

where $\sigma_{x x}^{a}$ and $\sigma_{x x}^{b}$ are the coefficients of the linear function. Consider the first rigid body virtual field describing a 
translation in the $x$ direction. Equation (26) can be substituted into Eq. (18) to obtain:

$$
\int_{-h_{s} / 2}^{h_{s} / 2}\left(\sigma_{x x}^{a}+\frac{y}{h_{s}} \sigma_{x x}^{b}\right) d y=\rho \int_{-h_{s} / 2}^{h_{s} / 2} \int_{0}^{x} a_{x} d x d y
$$

Evaluating the integrals and simplifying with the overline notation yields the coefficient $\sigma_{x x}^{a}$ :

$\sigma_{x x}^{a}=\rho x{\overline{a_{x}}}^{S}$

which is equivalent to the standard stress-gauge equation (Eq. 9). Now, consider the virtual field describing a rigid body rotation. Substituting Eq. (26) into Eq. (23) gives:

$$
\begin{aligned}
\int_{-h_{s} / 2}^{h_{s} / 2}\left(\sigma_{x x}^{a}+\frac{y}{h_{s}} \sigma_{x x}^{b}\right) y d y & -x \int_{-h_{s} / 2}^{h_{s} / 2} \sigma_{x y} d y \\
& =\rho \int_{-h_{s} / 2}^{h_{s} / 2} \int_{0}^{x}\left(a_{x} y-a_{y} x\right) d x d y
\end{aligned}
$$

Simplifying the integrals in Eq. (29) using the overline notation yields:

$\sigma_{x x}^{b} \frac{h_{s}}{12}-x{\overline{\sigma_{x y}}}^{y}=\rho x\left(\overline{a_{x} y}{ }^{S}-{\overline{a_{y}}}^{S}\right)$

The term ${\overline{\sigma_{x y}}}^{y}$ can be expressed in terms of acceleration averages using Eq. (21). Therefore, the coefficient $\sigma_{x x}^{b}$ can be given by:

$\sigma_{x x}^{b}=\frac{12 \rho x}{h_{s}}\left({\overline{a_{x} y}}^{S}-{\overline{a_{y}}}^{S}+x \bar{a}_{y}^{S}\right)$

Finally, a linear approximation of the stress distribution, $\sigma_{x x}(L S G)$, is obtained by combining the results for the two linear coefficients with Eq. (26):

$\sigma_{x x}(L S G)=\rho x{\overline{a_{x}}}^{S}+\frac{12 \rho x y}{h_{s}^{2}}\left({\overline{a_{x} y}}^{S}-{\overline{a_{y} x}}^{S}+x \bar{a}_{y}{ }^{S}\right)$

\section{References}

1. Prakash LJ (1995) Application of fine grained tungsten carbide based cemented carbides. Int J Refract Metals Hard Mater 13:257-264

2. Swab JJ, Meredith CS, Casem DT, Gamble WR (2017) Static and dynamic compression strength of hot-pressed boron carbide using a dumbbell-shaped specimen. J Mater Sci 52:10073-10084

3. Ravichandran G, Subhash G (1994) Critical appraisal of limiting strain rates for compression testing of ceramics in a split Hopkinson pressure bar. J Am Ceram Soc 77:263-267
4. Shih CJ, Meyers MA, Nesterenko VF, Chen SJ (2000) Damage evolution in dynamic deformation of silicon carbide. Acta Mater 48:2399-2420

5. Gama BA, Lopatnikov SL, Gillespie J, John W (2004) Hopkinson bar experimental technique: a critical review. Appl Mech Rev $57: 223-250$

6. Mandel K, Radajewski M, Krüger L (2014) Strain-rate dependence of the compressive strength of WC-Co hard metals. Mater Sci Eng A 612:115-122

7. Moulart R, Pierron F, Hallett SR, Wisnom MR (2010) Full-field strain measurement and identification of composites moduli at high strain rate with the virtual fields method. Exp Mech 51:509-536

8. Pierron E, Zhu H, Siviour C (2014) Beyond Hopkinson's bar. Philos Trans R Soc Lond A 2023:20130195

9. Zhu H (2015) A novel methodology for high strain rate testing using full-field measurements and the virtual fields methods. PhD, University of Troyes, France

10. Fletcher L, Van-Blitterswyk J, Pierron F (2018) A novel imagebased inertial impact (IBII) test for the transverse properties of composites at high strain rates. J Dyn Behav Mater (Submitted)

11. Pierron F, Forquin P (2012) Ultra-high-speed full-field deformation measurements on concrete spalling specimens and stiffness identification with the virtual fields method. Strain 48:388-405

12. Forquin P, Lukić B (2018) On the processing of spalling experiments. Part I: identification of the dynamic tensile strength of concrete. J Dyn Behav Mater 4:34-55

13. Lukić BB, Saletti D, Forquin P (2018) On the processing of spalling experiments. Part II: identification of concrete fracture energy in dynamic tension. J Dyn Behav Mater 4:56-73

14. Pierron F, Grédiac M (2012) The virtual fields method: extracting constitutive mechanical parameters from full-field deformation measurements. Springer, New York

15. Avril S, Grédiac M, Pierron F (2004) Sensitivity of the virtual fields method to noisy data. Comput Mech 34:439-452

16. Getting IC, Chen G, Brown JA (1993) The strength and rheology of commercial tungsten carbide cermets used in high-pressure apparatus. Pure Appl Geophys 141:545-577

17. Munro M (1997) Evaluated material properties for a sintered alpha-alumina. J Am Ceram Soc 80:1919-1928

18. Braiden PM, Davidge RW, Airey R (1977) Time-dependent strength parameters for tungsten carbides containing 6 or $16 \%$ cobalt at room and elevated temperatures. J Mech Phys Solids 25:257-268

19. Bhagat RB, Conway JC, Amateau MF, Brezler RA (1996) Tribological performance evaluation of tungsten carbide-based cermets and development of a fracture mechanics wear model. Wear 201:233-243

20. Han D, Mecholsky JJ (1990) Fracture analysis of cobalt-bonded tungsten carbide composites. J Mater Sci 25:4949-4956

21. Jewell P, Shannahan L, Pagano S, DeMott R, Taheri M, Lamberson L (2017) Rate and microstructure influence on the fracture behavior of cemented carbides WC-Co and WC-Ni. Int J Fract 208(1-2):203-219

22. Grédiac M, Blaysat B, Sur F (2017) A critical comparison of some metrological parameters characterizing local digital image correlation and grid method. Exp Mech 57:871-903

23. Piro J-L, Grédiac M (2004) Producing and transferring low-spatial-frequency grids for measuring displacement fields with moiré and grid methods. Exp Techn 28:23-26

24. Pierron F, Cherigéne R, Forquin P, Moulart R, Rossi M, Sutton MA (2011) Performances and limitations of three ultra high-speed imaging cameras for full-field deformation measurements. Appl Mech Mater 70:81-86

25. Rossi M, Pierron F, Forquin P (2014) Assessment of the metrological performance of an in situ storage image sensor ultra-high 
speed camera for full-field deformation measurements. Meas Sci Technol 25(2):025401

26. Grédiac M, Sur F, Blaysat B (2016) The grid method for in-plane displacement and strain measurement: a review and analysis. Strain 52:205-243

27. Herráez MA, Burton DR, Lalor MJ, Gdeisat MA (2002) Fast twodimensional phase-unwrapping algorithm based on sorting by reliability following a noncontinuous path. Appl Opt 41:7437-7444

28. Rossi M, Lava P, Pierron F, Debruyne D, Sasso M (2015) Effect of DIC spatial resolution, noise and interpolation error on identification results with the VFM. Strain 51:206-222
29. Lava P, Cooreman S, Coppieters S, De Strycker M, Debruyne D (2009) Assessment of measuring errors in DIC using deformation fields generated by plastic FEA. Opt Lasers Eng 47:747-753

30. Lava P, Cooreman S, Debruyne D (2010) Study of systematic errors in strain fields obtained via DIC using heterogeneous deformation generated by plastic FEA. Opt Lasers Eng 48:457-468

31. Rossi M, Pierron F (2012) On the use of simulated experiments in designing tests for material characterization from full-field measurements. Int J Solids Struct 49:420-435

32. Lukić B, Saletti D, Forquin P (2017) Use of simulated experiments for material characterization of brittle materials subjected to high strain rate dynamic tension. Philos Trans R Soc A 375:20160168 